\title{
Therapeutic Drug Monitoring of Antiepileptic Drugs in Epilepsy: A 2018 Update
}

\author{
Philip N. Patsalos, FRCPath, PhD \\ Epilepsy Society \\ Therapeutic Drug Monitoring Unit \\ Chalfont Centre for Epilepsy \\ Chalfont St Peter
}

\author{
AND \\ Department of Clinical and Experimental Epilepsy \\ UCL-Institute of Neurology \\ Queen Square, London, UK \\ Edgar P. Spencer, CChem, FRSC, PhD \\ Epilepsy Society \\ Therapeutic Drug Monitoring Unit \\ Chalfont Centre for Epilepsy \\ Chalfont St Peter
}

Correspondence to:

Professor Philip N. Patsalos

Therapeutic Drug Monitoring Unit

Chalfont Centre for Epilepsy

Chalfont St Peter

Buckinghamshire, SL9 0RJ

United Kingdom

Tel No.: 01494601355

Fax No.: 01494875176

E-mail:Philip.Patsalos@epilepsysociety.org.uk 


\section{Abstract}

Antiepileptic drugs (AEDs) are the mainstay of epilepsy treatment and since 198918 new AEDs have been licensed for clinical use and there are now 27 licensed AEDs in total for the treatment of patients with epilepsy. Furthermore, several AEDs are also used for management of other medical conditions e.g. pain and bipolar disorder. This has led to an increasingly widespread application of therapeutic drug monitoring (TDM) making AEDs among the most common medications for which TDM is performed. Indeed, TDM provides a pragmatic approach to epilepsy care in that bespoke dose adjustments are undertaken based on drug concentrations so as to optimize clinical outcome. For the older first generation AEDs (carbamazepine, ethosuximide, phenobarbital, phenytoin, primidone and valproic acid) much data has accumulated in this regard. However, increasingly this is occurring for the new AEDs (eslicarbazepine acetate, felbamate, gabapentin, lacosamide, lamotrigine, levetiracetam, oxcarbazepine, perampanel, piracetam, pregabalin, rufinamide, stiripentol, tiagabine, topiramate, vigabatrin and zonisamide). The aim of this review is to provide an overview of the indications for AED TDM, to provide key information for each individual AED in terms of the drug's prescribing indications, key pharmacokinetic characteristics, associated drug-drug pharmacokinetic interactions and the value and the intricacies for TDM for each AED. The concept of the reference range is discussed and also practical issues such as choice of sample type (total vs free concentrations in blood vs saliva) and sample collection and processing.

Abstract length: 235 words

Key words: antiepileptic drugs, therapeutic drug monitoring, pharmacokinetics, drug-drug interactions, saliva

Length: 11,000 words (includes headers and reference numbering)

Tables: 4 


\section{Introduction}

The introduction of 18 new antiepileptic drugs (AEDs) since 1989 has led to increasingly widespread application of therapeutic drug monitoring (TDM) in the clinical management of patients with epilepsy. Indeed, there are now 27 licensed AEDs (Table 1) making these drugs among the most common medications for which TDM is performed. ${ }^{1}$

TDM provides a pragmatic approach to epilepsy care in that bespoke dose adjustments can be undertaken guided by drug concentrations so as to optimize clinical outcome. Ideally AED treatment entails achieving complete seizure freedom without significant adverse effects, but for many patients achieving optimum seizure control with minimal adverse effects is the best compromise. Plasma or serum are the matrixes of choice for TDM but increasingly non-stimulated saliva is used. ${ }^{2}$ That AED TDM has become so widespread, and a significant adjunct to the treatment of epilepsy, can be attributed to the fact that for an individual patient it is difficult to identify the optimal dose on clinical grounds alone. There are several reasons for this including:- 1) Plasma AED concentrations correlate much better than dose with the clinical effects; 2) Assessment of therapeutic response on clinical grounds alone is difficult in most cases because AED treatment is prophylactic and seizures occur at irregular intervals. It is thus difficult to ascertain whether the prescribed dose will be sufficient to produce long-term seizure control; 3) It is not always easy to recognize signs of toxicity purely on clinical grounds; 4) AEDs are subject to substantial pharmacokinetic variability and thus large differences in dosage are required in different patients; and 5) There are no laboratory markers for clinical efficacy or toxicity of AEDs.

The aim of this review is to provide an overview of the indications for AED TDM, to provide key information for each AED in terms of the drug's prescribing indications in epilepsy and in non-epilepsy conditions (and non-licensed use), key pharmacokinetic characteristics, associated drug-drug pharmacokinetic interactions and the value and the intricacies for TDM for each AED. We briefly discuss practical issues such as choice of sample type and sample collection and processing; all being critical in allowing appropriate interpretation of TDM data. Because the reference range is much misunderstood, we begin by discussing this very important concept. 


\section{The reference range}

A major misconception in AED TDM is the meaning and interpretation of the reference range. The reference range is not a therapeutic range, instead it is defined as a range of drug concentrations which is quoted by a laboratory and specifies a lower limit below which a therapeutic response is relatively unlikely to occur, and an upper limit above which toxicity is relatively likely to occur. The reference range is based on population data derived from TDM research or from clinical drug trials. Because of large interindividual differences in type of epilepsy and severity of seizures, the effective AED concentration can vary significantly from patient to patient. Consequently, patients can achieve therapeutic benefit at plasma concentrations outside these ranges and it is not unusual for some patients to have optimum seizure control at plasma concentrations below the lower value of the reference range whilst others may require (and tolerate) drug concentrations above the upper limit of the reference range. ${ }^{1,3}$ Whilst adjusting drug dosages to achieve plasma concentrations within the reference range should be the initial goal, until the clinical response to the therapy can be assessed, it should be emphasized that it is important to treat the patient and not the blood concentration. ${ }^{4}$ It is thus inappropriate to adjust dosage to achieve blood concentrations within the reference range without taking into consideration the clinical status of the patient.

More recently, the concept of the "individual therapeutic concentration/range" has been proposed as the ideal practice parameter for bespoke AED therapy ${ }^{1}$ and is defined as the concentration, or range of concentrations, which has been empirically found to be associated with an optimum response in an individual patient. Identification of the individual therapeutic concentration can be very useful in clinical management because it provides a useful reference value for informing management decisions should a modification in the clinical status of a patient occur over time. The advantage of this approach is that it can be applied to any AED, including AEDs that have not yet had their references ranges clearly defined. Establishing the individual therapeutic concentration is straightforward and entails determining, preferably on two occasions, the steady-state concentration once a patient has been stabilized on his/her optimum 
AED regimen.

\section{Indications for TDM of AEDs}

The main indications for AED TDM are summarized in Table 2. Below we discuss these in some detail and highlight those AEDs to which the various indications apply. It should be emphasized, however, that there are some indications which apply to all AEDs, regardless of their specific pharmacokinetic/pharmacodynamic characteristics and these include, identifying non-compliance and suspected drug-related toxicity, guiding management of AED overdose or status epilepticus and ascertaining bioavailability changes upon switching patients from one formulation to another; in particular switching from a branded to a generic or a generic to generic.

\section{Indication 1: Dose optimization on the initially prescribed AED}

Plasma concentration measurement of the initially prescribed AED is particularly valuable when the best therapeutic response has been achieved in an individual and maintained for a sufficient period of time to be confident that dosage has been optimized (ideally this would be seizure freedom but for many patients it would entail optimum seizure control with minimal and tolerated adverse effects). Determination of the plasma AED concentration at a standardized sampling time will identify the "individual therapeutic concentration", which is a useful point of reference to guide treatment if a change in response occurs requiring further follow-up. ${ }^{1} \mathrm{~A}$ major advantage of the "individual therapeutic concentration" approach is that it does not rely on fixed "reference ranges" and can be applied to all AEDs regardless of whether or not "reference ranges" have been clearly defined. In order to establish an individual's therapeutic concentration, two separate determinations should be undertaken 2 to 4 months apart in order to estimate the extent of any variability i.e. the measured concentration values should be similar. ${ }^{1}$ Dosing strategies for AEDs towards improved personalized medicine would include increased application of TDM for more AEDs; progress in analytical methodology; advances in pharmacogenomics; increased use of genotyping for AEDs and increased focus on special patient groups. ${ }^{5}$

\section{Indication 2: Uncontrolled seizures}


Knowledge of the "individual therapeutic concentration" will improve the management of patients who develop breakthrough seizures after a prolonged period of seizure control. For example, after a breakthrough seizure occurs, if the plasma concentration is much lower than the previously determined individual therapeutic concentration it suggests either suboptimal compliance ${ }^{6}$ or a clinically important change in AED pharmacokinetics. ${ }^{7-9}$ In a setting where seizures persist despite an apparently adequate dosage of an appropriate $A E D, T D M$ is useful to identify potential causes of therapeutic failure which may result from poor compliance ${ }^{6}$ (typically characterized by variable plasma concentrations, which increase following supervised drug intake) or from poor drug absorption, fast metabolism or drug interactions (typically characterized by low plasma AED concentrations).

\section{Indication 3: Suspected toxicity}

Suspected toxicity is one of the most frequent reasons why AED measurements are requested and the most common adverse effects are CNS-related and include fatigue, irritability, coordination difficulties, sedation, dizziness, confusion, drowsiness, tremor and nystagmus. ${ }^{10}$ All AEDs act as CNS depressants and in particular when polydrug therapy is required, the determination of all prescribed AEDs may help to identify which drug(s), if any, are contributing to suspected CNS toxicity. Furthermore, elevated plasma concentrations of many AEDs can exacerbate seizures and urgent TDM will assist the differential diagnosis in the case of a known epileptic patient admitted with seizures. Finally, cases of suspected acute overdose require TDM both for the initial diagnosis and also for timing reinstatement of the drug regimen so as to maintain seizure control if the patient has epilepsy and taken an overdose of own AED medication.

Both plasma and for many AEDs, salivary drug concentrations, can aid in confirming a diagnosis of drug toxicity. ${ }^{1,2}$ Furthermore, AED monitoring is particularly valuable in patients whose clinical status is difficult to assess e.g. young children and subjects with associated mental and/or physical disability. Salivary measurements may be particularly appropriate in this setting e.g. in a series of 175 patients with epilepsy and mental disability, phenytoin and carbamazepine salivary concentrations exceeded the drug reference ranges on numerous occasions and the authors suggest that regular salivary 
TDM, along with neurological assessments be undertaken in order to avoid toxic drug concentrations in this population. ${ }^{11}$ The pharmacokinetics of carbamazepine in saliva and plasma were recently observed to be similar in a series of 20 patients admitted to an Emergency Toxicology Unit with acute poisoning from carbamazepine. ${ }^{12}$ The authors conclude that saliva carbamazepine monitoring can be usefully applied in managing such patients.

\section{Indication 4: Children}

Age markedly influences AED pharmacokinetics and plasma clearance of AEDs in children, and is significantly higher than adults. ${ }^{13-15} \mathrm{~A}$ child may need a 2-3 times greater weight for weight dose than that required to achieve the same plasma drug concentration in an adult. Furthermore, clearance decreases gradually throughout childhood, but the precise time course of this process is not well established and is characterized by pronounced inter-individual variability. ${ }^{13}$ Dose requirements for children are less predictable than for adults and constantly changing, therefore TDM is particularly helpful for optimal management of this patient group. ${ }^{14,16}$ Furthermore, most new AEDs are not initially approved for use in children but some clinicians may prescribe them off label in difficult cases provided TDM is available. Since there is a greater need to monitor AED concentrations in children, there have been many studies of salivary TDM in this patient group ${ }^{17-19}$ which generally conclude that saliva is often acceptable and preferred to blood sampling in children. This approach may be particularly valuable for preterm infants whose clinical situation is very fragile and may require repeated TDM; indeed, this procedure was recently described for caffeine monitoring. ${ }^{20}$

\section{Indication 5: Pregnancy}

The teratogenic effects of AEDs are well documented. ${ }^{21-29}$ Nevertheless, AEDs continue to be prescribed through pregnancy so as to maintain seizure control and not compromise the well-being of the foetus consequent to maternal seizure activity. An important part of the care of pregnant women with epilepsy includes folic acid supplementation and regular monitoring of their AEDs before, during and after the pregnancy, including measurement of AED concentrations in mammary milk when breast-feeding is occurring. 
Many physiological changes occur during pregnancy which alter drug pharmacokinetics and AED dose requirements. ${ }^{21-26,30-32}$ Careful and frequent adjustments to drug dosage may be required throughout pregnancy in order to improve the efficacy and safety of the prescribed medication. Gastrointestinal function is often prolonged by a decrease in gut motility (particularly during the third trimester) and as gestation advances the quantity of total body water and fat increases which affects the volume of distribution causing plasma drug concentrations to decrease. Changes in cardiac output, ventilation, and renal/hepatic blood flow also occur which affect drug elimination; furthermore, plasma protein concentration decreases which causes the free (pharmacologically effective) drug concentration to increase. Renal blood flow and glomerular filtration rate also change which affects, in particular, the plasma concentrations of those drugs predominantly eliminated unchanged by the kidneys. The increased secretion of estrogen and progesterone in pregnancy can alter the metabolizing capacity of hepatic enzymes; in addition the placental transport of drugs and their compartmentalization in the embryo/placenta along with metabolism by the placenta/fetus can play an important role in modifying the pharmacokinetics of AEDs during gestation.

In summary, the pharmacokinetics of many AEDs are altered by pregnancy which usually causes plasma concentrations to decline (sometimes rapidly) throughout gestation but the extent of this effect varies with different AEDs and between patients. ${ }^{33}$ Thus decreases in plasma AED concentration may be insignificant in some patients and pronounced in others who may require dosage adjustments to maintain seizure control. Monitoring AED concentrations regularly, particularly for lamotrigine, levetiracetam, oxcarbazepine, phenytoin, carbamazepine and valproate ${ }^{34}$ in either plasma or saliva is recommended during pregnancy ${ }^{33}$ and the value of salivary monitoring has been well documented ${ }^{2}$ and may be particularly valuable for those drugs where the concentration in saliva reflects the non-protein bound quantity in plasma and/or when frequent TDM is recommended.

\section{Indication 6: Elderly}

Advancing age alters not only the disposition of drugs but also the way the body responds 
to them. ${ }^{13,15,35}$ There is substantial inter- and intra-individual variation in the pharmacokinetic changes that occur with advancing age which results in large differences in the relationship between drug dose and plasma concentration. ${ }^{15}$ The greater pharmacodynamic sensitivity in the elderly, affects their response to a given plasma concentration which, in turn, complicates interpretation of TDM results. ${ }^{36}$ Once again the concept of an "individual therapeutic concentration" is important and TDM of AEDs in the elderly is particularly helpful for identifying sub-optimal compliance e.g. under-dosing, overdosing, missed doses or make-up doses which are common in older patients and affect plasma drug concentrations and potentially the clinical response. Drug polytherapy is significantly increased in the elderly compared to other age groups, particularly with drugs required for treating co-morbidities and recently it has been reported that up to nine concomitant CNS-active drugs are often prescribed. ${ }^{37}$ Therefore, drug-drug pharmacokinetic interactions are more likely to occur in the elderly and TDM is a valuable procedure for monitoring these. ${ }^{35}$ Albumin concentrations are also decreased in older age and measurement of non-protein bound free drug concentration is particularly useful, especially for extensively protein bound AEDs. ${ }^{38}$ Salivary monitoring could also be helpful. ${ }^{2}$

\section{Indication 7: Co-morbidities}

Many co-morbidities/co-pathologies e.g. hepatic or renal impairment, infections, burns, stroke, decreased cardiac function, HIV infection and other conditions can significantly affect the absorption, distribution, elimination and protein binding of AEDs. ${ }^{39-43}$ In addition to the alterations caused by the pathological state per se, drugs used to treat these conditions can cause drug-drug pharmacokinetic interactions and consequent changes in AED concentrations which can be monitored by TDM.

Whenever a concurrent condition is known or suspected to alter AED protein binding e.g. renal failure and after dialysis or surgery, when hypoalbuminemia occurs, or when patients receive drugs that compete for protein binding sites e.g. aspirin, naproxen, tolbutamide, phenylbutazone,${ }^{44}$ measurement of the free drug concentration is essential, particularly for extensively bound AEDs.

Hepatic disease can significantly alter the elimination of AEDs that are metabolized in the 
liver; 45 furthermore, since the liver is the source of many proteins, plasma protein binding may also be affected in patients with liver disease. Since it is impossible to predict the extent of change in AED clearance in hepatic disease ${ }^{45}$ TDM (with free concentrations for highly bound drugs) is considered best practice in this patient group.

\section{Indication 8: Drug-drug pharmacokinetic interactions}

Approximately $30 \%$ of patients with epilepsy are refractory to monotherapy and are invariably prescribed two or more AEDs in order to control their seizures. ${ }^{46-49}$ In addition, because of the long-term nature of epilepsy treatment, often for life, it is inevitable that patients will be prescribed non-epilepsy drugs to treat co-morbidities. In both these settings the propensity of drug-drug pharmacokinetic interactions is high and can result in either an increase or a decrease in plasma AED concentrations. ${ }^{50}$ Thus if patients exhibit signs of toxicity or experience breakthrough seizures, AED TDM can help ascertain which drug is responsible for the change in clinical status and also help guide dosage adjustments so as to compensate for the interaction. ${ }^{47,50}$ Best practice is to avoid interacting drugs, but if this is not possible, it is advisable to measure baseline concentrations of on-going therapy prior to making the addition. Both plasma and salivary monitoring can be used to quantify pharmacokinetic interactions and saliva may be particularly appropriate to investigate a plasma protein binding displacement interaction that can occur with highly protein bound AEDs such as phenytoin and valproic acid. ${ }^{2}$

\section{Indication 9: Switching to another AED formulation and generic substitution}

Most AEDs are available in a variety of formulations some of which are not interchangeable e.g. immediate-release versus sustained-release formulations. It is good practice therefore to determine AED concentrations before and after a formulation change since this will identify differences in bioavailability that alter steady-state concentrations and perhaps cause a change in the clinical status of the patient. ${ }^{51-53}$

In addition, many AEDs are now available as generic formulations and even though they are considered bioequivalent to the branded product there are various reports that a significant minority of patients experience breakthrough seizures when switched from a branded to a generic formulation. ${ }^{54-56}$ Furthermore, switching from a generic to a generic 
may result in a greater incidence of breakthrough seizures. ${ }^{57}$ However, more recent studies which involved patients taking lamotrigine and with a confirmed history of having potential problems with generic switching, not only were the two generic products determined to be bioequivalent but, in addition, there were no significant changes in seizure control or adverse effects. ${ }^{58,59}$ Nevertheless, it is good practice to determine plasma AED concentrations before and after the switch so as to definitively ascertain that a difference in bioavailability caused the change in clinical status of the patient.

\section{Individual AEDs}

In the following section the AEDs will be reviewed in alphabetical order with regards to their clinical indications, key pharmacokinetic properties (Table 3), drug-drug pharmacokinetic interaction profiles and their TDM characteristics (Table 4).

\section{Brivaracetam}

Indications: Brivaracetam is indicated as adjunctive therapy in the treatment of partialonset seizures with or without secondary generalization in adult and adolescent patients from 16 years of age with epilepsy.

Pharmacokinetics: Brivaracetam exhibits linear pharmacokinetics. After oral ingestion It is rapidly absorbed with a time to maximum plasma concentration (Tmax) of $0.5-1.0 \mathrm{~h}$, a bioavailability of $100 \%$ and a volume of distribution $(\mathrm{Vd})$ of $0.5 \mathrm{~L} / \mathrm{kg} .{ }^{60}$ The plasma protein binding of brivaracetam is $35 \% .{ }^{61}$ Brivaracetam undergoes extensive (>90\%) hepatic metabolism via hydrolysis of its acetamide group, via an amidase, and secondary hydroxylation through the CYP2C19 isoenzyme pathway. The 3 major metabolites carboxylic acid, hydroxy, and hydroxyacid are not pharmacologically active. ${ }^{62,63}$ The plasma elimination half-life ( $t / 1 / 2)$ of brivaracetam is $7-8 \mathrm{~h}$.

Drug-drug pharmacokinetic interactions: Because brivaracetam undergoes metabolism primarily via CYP-independent hydrolysis, it has low interaction potential; however, brivaracetam plasma concentrations are decreased when co-administered with the enzyme inducing AEDs carbamazepine, phenobarbital and phenytoin. ${ }^{64}$ Also, rifampin can decrease brivaracetam concentrations.

Brivaracetam TDM: Because of the narrow therapeutic index and wide inter-individual 
variability in the rate of elimination, TDM of brivaracetam for patients receiving therapy is essential particularly when co-medicated with drugs which affect brivaracetam plasma concentrations. The monitoring of plasma concentration is also indicated for compliance testing, ascertaining toxicity and also for elucidating clinical interactions. The current reference range for brivaracetam in plasma is $0.2-2.0 \mathrm{mg} / \mathrm{L} .{ }^{65}$ Brivaracetam distributes into saliva and concentrations reflect the non-protein bound concentration in plasma; thus, saliva can be a useful alternative matrix for brivaracetam TDM. ${ }^{66}$

\section{Carbamazepine}

Indications: Carbamazepine is a first line drug for the treatment of partial and secondarily generalized tonic-clonic seizures and also primary generalized tonic-clonic seizures. It is also used to treat trigeminal neuralgia and bipolar disorder unresponsive to lithium. Pharmacokinetics: The pharmacokinetics of carbamazepine are non-linear because of autoinduction which completes within 3 weeks and can result in a 3-fold increase in elimination. ${ }^{67,68}$ Following oral ingestion the absorption of carbamazepine is erratic and variable with Tmax being formulation-dependent (range $0.5-9.0 \mathrm{~h}),{ }^{69}$ bioavailability is 75 $85 \%$ and $\mathrm{Vd}$ is $0.8-2.0 \mathrm{~L} / \mathrm{kg}$. Carbamazepine is $75 \%$ bound to plasma proteins and its pharmacologically active metabolite, carbamazepine-epoxide, is $50 \%$ protein bound. ${ }^{61}$ Carbamazepine is extensively metabolized in the liver, primarily by CYP3A4, to carbamazepine-epoxide which accumulates in plasma and is pharmacologically equipotent to carbamazepine. Carbamazepine-epoxide is further metabolized, by epoxide hydrolase, to the pharmacologically inactive 10,11-diol which is eliminated in urine partly unchanged and partly as a glucuronide conjugate. The $t_{1} / 2$ of carbamazepine in adults is $8-20 \mathrm{~h}$, whilst in children it is $10-13 \mathrm{~h}$ and the elderly 30-50 h. The $\mathrm{t}_{1 / 2}$ of carbamazepine-epoxide is $\sim 34 \mathrm{~h}$. Drug-drug pharmacokinetic interactions: The metabolism of carbamazepine via CYP3A4 is readily inducible and also potently inhibited; therefore it is subject to many pharmacokinetic drug-drug interactions. The AEDs that inhibit the metabolism of carbamazepine and thus increase carbamazepine blood concentrations include: clobazam and stiripentol. In contrast, felbamate, oxcarbazepine, phenobarbital, phenytoin, primidone and rufinamide induce carbamazepine metabolism and decrease carbamazepine concentrations. ${ }^{70}$ 
Carbamazepine metabolism can also be affected by many non-epilepsy drugs. The drugs that inhibit carbamazepine metabolism and increase blood concentrations include: clarithromycin, cimetidine, ciprofloxacin, danazol, diltiazem, erythromycin, fluconazole, fluoxetine, flurithromycin, grapefruit juice, haloperidol, isoniazid, isotretinoin, josamycin, ketoconazole, metronidazole, miconazole, nefazodone, nelfinavir, nicotinamide, ponsinomycin, propoxyphene, ritonavir, ticlopidine, trazodone, troleandomycin, verapamil and viloxazine. Non-epilepsy drugs that induce carbamazepine metabolism and decrease blood concentrations include: efavirenz, probenecid, rifampicin, risperidone, St John's Wort and theophylline. Some drugs do not alter carbamazepine concentrations per se, but can increase carbamazepine-epoxide concentrations, via inhibition of epoxide hydrolase, and may cause typical carbamazepine toxicity. These drugs include brivaracetam, valproic acid, zonisamide, amoxapine, loxapine and quetiapine. ${ }^{70,71}$

Carbamazepine TDM: Since the pharmacokinetics of carbamazepine are non-linear (due to autoinduction), that its efficacy and adverse effects can in part be attributable to its pharmacologically active metabolite carbamazepine-epoxide and the fact that it is the target of many drug-drug pharmacokinetic interactions, results in large differences between individuals in the dose to plasma concentration relationship. All of these factors make TDM particularly useful during carbamazepine therapy; furthermore, there is considerable interpatient variability in the carbamazepine concentration that is associated with an optimal therapeutic response (which may in part be due to the variation in carbamazepine-epoxide concentration). In practice both carbamazepine and carbamazepine-epoxide concentrations should be monitored. The current reference range for carbamazepine in plasma is $4-12 \mathrm{mg} / \mathrm{L}$ whilst that of carbamazepine-epoxide is up to $2.3 \mathrm{mg} / \mathrm{L}{ }^{1}{ }^{1}$ Carbamazepine and carbamazepine-epoxide may also be determined in saliva where the salivary concentration of each is similar to the pharmacologically active, non-protein bound free concentration in plasma. ${ }^{2}$

\section{Clobazam}

Indications: Clobazam is licensed for the adjunctive intermittent treatment of partial seizures or generalized seizures in patients $>3$ years old and also for the management of 
non-convulsive status epilepticus. Non-licensed use includes the treatment of various epilepsies (e.g. reading, catamenial, startle and benign childhood partial epilepsy); also febrile seizures and seizures associated with alcohol withdrawal.

Pharmacokinetics: Clobazam exhibits linear pharmacokinetics. After oral ingestion it is rapidly absorbed with a Tmax of $1-3 \mathrm{~h}$, a bioavailability of $>95 \%$. and a $\mathrm{Vd}$ is $0.87-1.83$ $\mathrm{L} / \mathrm{kg}$. The plasma protein binding of both clobazam and its pharmacologically active metabolite, $\mathrm{N}$-desmethyl clobazam, is $90 \%{ }^{61}$ Clobazam is extensively metabolized in the liver, primarily by demethylation, to a pharmacologically active metabolite $\mathrm{N}$-desmethyl clobazam together with some 4-hydroxy metabolites. N-desmethyl clobazam can accumulate in plasma to higher concentrations than the parent drug and is responsible for much of the clinical effect ${ }^{72}$ and is subsequently metabolized by CYP2C19 to 4-hydroxydesmethylclobazam. The $\mathrm{t}_{1 / 2}$ of clobazam in adults is $10-30 \mathrm{~h}$, whilst that of $\mathrm{N}$-desmethyl clobazam is $36-46 \mathrm{~h}$. In children clobazam $\mathrm{t} / 2$ is $\sim 16 \mathrm{~h}$ whilst in the elderly it is $30-48 \mathrm{~h}$. Drug-drug pharmacokinetic interactions: The metabolism of clobazam, primarily via CYP3A4 but also via CYP2C19, can be readily induced or inhibited and consequently various drug-drug pharmacokinetic interactions have been described. The AEDs that inhibit the metabolism of clobazam and thus increase clobazam blood concentrations include: felbamate and stiripentol. ${ }^{70}$ In contrast, carbamazepine, phenobarbital and phenytoin induce clobazam metabolism and decrease clobazam concentrations. Etravirine, ketoconazole, miconazole and omeprazole can inhibit the metabolism of clobazam resulting in increases in blood concentrations of both clobazam and $\mathrm{N}$ desmethylclobazam. ${ }^{70}$

Clobazam TDM: The presence of the pharmacologically active metabolite of clobazam, $\mathrm{N}$ desmethylclobazam, complicates the therapeutics of clobazam because it is not only present in blood at much greater concentrations than the parent drug but also its metabolism, via CYP2C19, is under polymorphic control so that some patients have little ability to metabolize it. Indeed, there are reports of excessive accumulation of $\mathrm{N}$ desmethylclobazam with associated toxicity in patients with a low activity of CYP2C19. ${ }^{73}$ Consequently there are large differences between individuals in the dose to plasma concentration relationship and it is essential that TDM entails the simultaneous measurement of both clobazam and $\mathrm{N}$-desmethylclobazam. The current reference range 
for clobazam in plasma is $0.03-0.3 \mathrm{mg} / \mathrm{L}$ whilst that of $\mathrm{N}$-desmethylclobazam is $0.3-3.0$ $\mathrm{mg} / \mathrm{L} .{ }^{1}$ Clobazam and $\mathrm{N}$-desmethylclobazam distribute into saliva and it has been demonstrated in children that salivary concentrations are significantly correlated with those in plasma; thus saliva can be used as an alternative matrix for clobazam TDM. ${ }^{2}$

\section{Clonazepam}

Indications: Clonazepam is licensed for the treatment of a variety of seizure types including absence, akinetic, atonic and myoclonic seizures. Clonazepam is also licensed for the treatment of Lennox-Gastaut syndrome and the management of status epilepticus. Nonlicensed use includes the treatment of acquired epileptic aphasia (Landau-Kleffner syndrome), infantile spasms (West syndrome) and neonatal seizures.

Pharmacokinetics: Clonazepam exhibits linear pharmacokinetics. After oral ingestion clonazepam is rapidly absorbed with a Tmax of $1-4 \mathrm{~h}$ and a bioavailability of $>80 \%$. It is $90 \%$ bound to plasma proteins and its $\mathrm{Vd}$ is $1.5-4.4 \mathrm{~L} / \mathrm{kg} .{ }^{61}$ Clonazepam is extensively metabolized in the liver, via CYP3A4, to produce 7-amino-clonazepam which is partially acetylated to form 7 -acetamido-clonazepam. The $t / 2$ of clonazepam in healthy adults is 17 $56 \mathrm{~h}$ whilst in children it is $23-33 \mathrm{~h}$ and in neonates is $22-81 \mathrm{~h}$.

Drug-drug pharmacokinetic interactions: The metabolism of clonazepam can be both inhibited and induced but there are few examples of drug-drug pharmacokinetic interactions. Thus, the AEDs carbamazepine, phenobarbital, phenytoin and primidone enhance its metabolism and decreases plasma clonazepam concentrations whilst felbamate inhibits its metabolism and increases clonazepam concentrations. ${ }^{70}$ There are no other drug-drug pharmacokinetic interactions reported with clonazepam.

Clonazepam TDM: Clonazepam elimination is associated with significant inter-individual variability and consequent large differences between individuals in the dose to plasma concentration relationship. In children with absence seizures, efficacy was reported in the plasma concentration range $13-72 \mu \mathrm{g} / \mathrm{L}^{74}$ and in neonates receiving intravenous clonazepam for convulsions; efficacy was achieved at plasma concentrations of 28-117 $\mu \mathrm{g} / \mathrm{L} .{ }^{75}$ The current reference range for clonazepam in plasma is $0.02-0.07 \mathrm{mg} / \mathrm{L} .{ }^{1}$ The secretion of clonazepam into saliva has not been investigated. ${ }^{2}$ 


\section{Diazepam}

Indications: Diazepam is licensed for the management of febrile convulsions and of status epilepticus. It is also licensed as an anxiolytic, a skeletal muscle relaxant and as a perioperative sedative and analgesic.

Pharmacokinetics: Diazepam is rapidly absorbed after intramuscular (IM), rectal and oral absorption with a Tmax of 10-60 minutes, 30-60 minutes and 30-90 minutes respectively. ${ }^{76-78}$ Bioavailability by oral and rectal routes is generally $>95 \%, \mathrm{Vd}$ is $1-2 \mathrm{~L} / \mathrm{kg}$ and plasma protein binding is $98 \%$. Diazepam is extensively metabolized in the liver, primarily by demethylation, to a pharmacologically active metabolite $\mathrm{N}$ desmethyldiazepam. Both diazepam and N-desmethyl-diazepam are further metabolised to produce temazepam and oxazepam respectively. The demethylation and hydroxylation reactions are catalyzed by CYP3A4 and CYP2C19. N-desmethyldiazepam can accumulate in plasma to higher concentrations than the parent drug and is responsible for much of the clinical effect. ${ }^{79}$ The $t \frac{1}{2}$ of diazepam in adults is $24-48 \mathrm{~h}$, whilst that of $\mathrm{N}$ desmethyldiazepam it is 72-96 $\mathrm{h}$. In children diazepam $\mathrm{t} / 2$ is $\sim 18 \mathrm{~h}$ whilst in the elderly it is prolonged and age related.

Drug-drug pharmacokinetic interactions: Diazepam is subject to some drug-drug pharmacokinetic interactions. The enzyme-inducing AEDs carbamazepine, phenobarbital, phenytoin and primidone enhance diazepam metabolism and decrease diazepam and $\mathrm{N}$ desmethyl diazepam plasma concentrations whilst cimetidine, erythromycin, fluvoxamine, itraconazole and omeprazole can inhibit its metabolism and increase diazepam plasma concentrations. Valproic acid can displace diazepam from its plasma protein biding sites and also inhibit its metabolism so that free diazepam concentrations are increased whereas $\mathrm{N}$-desmethyl diazepam concentrations are decreased. ${ }^{80}$

Diazepam TDM: Diazepam plasma concentrations are not routinely used to guide patient management. The presence of the pharmacologically active metabolite of diazepam, $\mathrm{N}$ desmethyldiazepam, complicates the therapeutics of diazepam because in patients maintained on diazepam it is not only present in blood at much greater concentrations than the parent drug but also its metabolism, via CYP2C19, is under polymorphic control so that some patients have little ability to metabolize it. Furthermore, many patients develop tolerance to the anti-seizure effects of diazepam. Consequently, there are large 
differences between individuals in both the dose to plasma concentration relationship and also the plasma concentration to clinical effect relationship. The maximum plasma concentration required to suppress seizures depends on various clinical factors including seizure type, and duration of therapy and ranges $200-600 \mathrm{ng} / \mathrm{mL}$ in most emergency settings. ${ }^{81}$ For initial seizure control, plasma diazepam concentrations of $550 \mathrm{ng} / \mathrm{mL}$ are suggested, whilst for maintenance of seizure control, plasma concentrations of 150-300 $\mathrm{ng} / \mathrm{L}$ are suggested. ${ }^{76}$ Diazepam and $\mathrm{N}$-desmethyldiazepam distribute into saliva where concentrations reflect the non-protein bound plasma concentration. ${ }^{82}$

\section{Eslicarbazepine acetate}

Indications: Eslicarbazepine acetate is licensed for the adjunctive treatment of partial onset seizures with or without secondary generalization in patients with epilepsy aged 16 years and older. Non-licensed use includes the treatment of bipolar disorder, cranial neuralgia, headache, neuropathic pain and trigeminal neuralgia.

Pharmacokinetics: Eslicarbazepine acetate is a pro-drug for eslicarbazepine; the latter exhibiting linear pharmacokinetics. After oral ingestion eslicarbazepine acetate is rapidly absorbed so that Tmax for eslicarbazepine is $2-3 \mathrm{~h} .{ }^{83}$ Its bioavailability is $>90 \%$ and the $\mathrm{Vd}$ is $2.7 \mathrm{~L} / \mathrm{kg}$. Plasma protein binding is $44 \% .{ }^{61}$ Eslicarbazepine acetate is rapidly metabolized by esterases in the liver to its pharmacologically active metabolite eslicarbazepine (also known as S-licarbazepine, 10-hydroxycarbazepine and monohydroxy derivative). 10-hydroxycarbazepine is the racemic mixture of the pharmacologically active metabolite of oxcarbazepine. Eslicarbazepine (33\%) is subsequently metabolized by conjugation with glucuronic acid via UGT1A4, UGT1A9, UGT2B4, UGT2B7 and UGT2B17, the latter being principally involved. In addition, small amounts of two other pharmacologically active metabolites are formed from eslicarbazepine acetate (Rlicarbazepine [5\%] and oxcarbazepine [1\%]). The plasma $t \frac{1}{2}$ of eslicarbazepine in adults is 13-20 h.

Drug-drug pharmacokinetic interactions: The drug is subject to very few drug-drug pharmacokinetic interactions. Thus, carbamazepine, phenytoin and topiramate enhance its elimination and decrease eslicarbazepine plasma concentrations. ${ }^{70}$

Eslicarbazepine TDM: The current reference range for eslicarbazepine in plasma is 3-35 
$\mathrm{mg} / \mathrm{L}$ which is based on that for 10-hydroxycarbazepine derived from oxcarbazepine. ${ }^{1}$ The secretion of eslicarbazepine acetate into saliva has not been investigated. However, since the pharmacologically active metabolite, eslicarbazepine, is similar to $10-$

hydroxycarbazepine, it is likely to be secreted into saliva and interpretation of salivary concentrations would be similar to that of 10-hydroxycarbazepine. ${ }^{2}$

\section{Ethosuximide}

Indications: Ethosuximide is licensed for the monotherapy treatment of absence seizures in patients of all ages. Non-licensed use includes the treatment of astatic (drop attacks), atypical absence and myoclonic seizures.

Pharmacokinetics: Ethosuximide exhibits linear pharmacokinetics. After oral ingestion it is rapidly absorbed with a Tmax of $1-4 \mathrm{~h}$, a Vd of $0.7 \mathrm{~L} / \mathrm{kg}$. Its bioavailability is $>90 \%$ and it is $22 \%$ protein bound. ${ }^{84,85,61}$ Ethosuximide is extensively metabolized in the liver, primarily via CYP3A, to form various metabolites of which $>40 \%$ are glucuronide conjugates. The $t_{1 / 2}$ in adults of $40-60 \mathrm{~h}$ and in children it is 30-40 $\mathrm{h}$.

Drug-drug pharmacokinetic interactions: Metabolism of ethosuximide can be both induced and inhibited, but only a few drug-drug pharmacokinetic interactions are reported. Carbamazepine, phenobarbital, phenytoin, primidone and rifampicin enhance its elimination and decrease ethosuximide plasma concentrations. In contrast, stiripentol and isoniazid, and possibly valproic acid, inhibit the metabolism of ethosuximide and increase ethosuximide plasma concentrations. ${ }^{70}$

Ethosuximide TDM: The current reference range for ethosuximide in plasma is $40-100$ $\mathrm{mg} / \mathrm{L}$. The drug is also distributed into saliva at similar concentrations to those in plasma, thus saliva can be used as an alternative matrix for TDM. ${ }^{2}$

\section{Felbamate}

Indications: Felbamate is not licensed for use in the UK, although it is available on a named patient basis. Where licensed, its use is restricted to patients who do not respond adequately to alternative treatments and particularly those with partial and secondary generalized seizures or Lennox-Gastaut syndrome. These restrictions have been placed on the drug because it is associated with an increased risk of aplastic anaemia and 
hepatotoxicity. Non-licensed use includes the treatment of absence seizures, acquired epileptic aphasia, infantile spasms and juvenile myoclonic epilepsy.

Pharmacokinetics: The pharmacokinetics of felbamate are linear. Following oral ingestion it is rapidly absorbed with a Tmax of 2-6 hours, bioavailability is $>90 \%$, Vd is $0.7-0.91 \mathrm{~L} / \mathrm{kg}$ and it is $48 \%$ bound to plasma proteins. ${ }^{61}$ Approximately $50 \%$ of the dose is metabolized in the liver, via CYP3A4 and CYP2E1, to form p-hydroxy and 2-hydroxy felbamate (10$15 \%)$ together with a variety of unidentified metabolites, some of which are glucuronides. The hepatotoxicity and aplastic anaemia which occurs in a few patients treated with felbamate is due to the formation of a reactive atropaldehyde metabolite which is said to accumulate in some individuals and cause toxicity. ${ }^{86}$ The $t_{1 / 2}$ of felbamate in adults is $16-22$ h.

Drug-drug pharmacokinetic interactions: There are few drug-drug pharmacokinetic interactions affecting felbamate. Thus, carbamazepine and phenytoin enhance its elimination and decrease felbamate plasma concentrations whilst valproic acid inhibits its metabolism and increases felbamate plasma concentrations. ${ }^{70}$ Interestingly, gabapentin can increase plasma felbamate concentration by inhibiting its renal elimination. ${ }^{70}$

Felbamate TDM: The current reference range for felbamate in plasma is $30-60 \mathrm{mg} / \mathrm{L} .^{1,87}$ Monitoring of felbamate concentrations does not predict the rare adverse effects associated with felbamate treatment. The secretion of felbamate into saliva has not been investigated. $^{2}$

\section{Fosphenytoin}

Indications: Fosphenytoin is licensed for treatment of generalized convulsive status epilepticus and as a short-term substitute for oral phenytoin if oral administration is not possible and/or contraindicated. It is also licensed for use to prevent seizures occurring during neurosurgery and/or head trauma.

Pharmacokinetics: Fosphenytoin is a pro-drug for phenytoin; the latter exhibiting non-linear pharmacokinetics. Following IM administration, fosphenytoin is rapidly absorbed with a Tmax of $\sim 0.5 \mathrm{~h}$ and bioavailability of $100 \%$. Phenytoin Tmax is $0.5-1.0 \mathrm{~h}$ following the start of an intravenous infusion of fosphenytoin and 1.5-4.0 h following intramuscular administration. The $t / 2$ for conversion of fosphenytoin to phenytoin is $8-15$ minutes. ${ }^{10}$ The 
pharmacokinetic characteristics for derived phenytoin are exactly those observed when phenytoin is administered directly (see phenytoin section).

Drug-drug pharmacokinetic interactions: No drugs are reported to interfere with the conversion of fosphenytoin to phenytoin. Drug-drug pharmacokinetic interactions following fosphenytoin administration are those expected to occur with drugs known to interact with phenytoin (see phenytoin section).

Fosphenytoin TDM: Fosphenytoin plasma concentrations are not necessary or helpful to guide patient management; however, phenytoin TDM is invaluable (see phenytoin section).

\section{Gabapentin}

Indications: Gabapentin is approved for the monotherapy treatment of partial seizures with or without secondary generalization in adults and children aged 12 years and above; also as adjunctive therapy in adults and children aged 6 years and above. In addition, the drug is licensed for the treatment of peripheral neuropathic pain. Non-licensed use includes the treatment of anxiety, bipolar disorder, phantom limb pain and trigeminal neuralgia.

Pharmacokinetics: The pharmacokinetics of gabapentin are non-linear because the drug is absorbed in the proximal small bowel by the L-amino acid transporter which is a saturable mechanism. Following oral ingestion gabapentin is rapidly absorbed with a Tmax of 2-3 hours and a bioavailability of $<60 \%$. It is not protein bound and its $\mathrm{Vd}$ is $0.65-1.94 \mathrm{~L} / \mathrm{kg}$. ${ }^{61}$ Gabapentin is not metabolized, being cleared entirely by renal elimination with a $t \frac{1}{2}$ in adults with normal renal function of 5-9 h. ${ }^{88}$

Drug-drug pharmacokinetic interactions: Gabapentin is not subject to drug-drug pharmacokinetic interactions with other AEDs. ${ }^{89}$ However, hydrocodone, cimetidine, morphine and naproxen can increase gabapentin plasma concentrations whilst antacids can decrease concentrations. ${ }^{70}$

Gabapentin TDM: That gabapentin is associated with non-linear pharmacokinetics, due to variable absorption, resulting in large differences between individuals in the dose to plasma concentration relationship, makes it an excellent candidate for TDM. The current reference range for gabapentin in plasma is $2-20 \mathrm{mg} / \mathrm{L} .{ }^{1}$ Gabapentin is secreted into saliva; however concentrations are much lower than those observed in plasma, with a significant correlation between saliva and plasma concentrations. ${ }^{2}$ 


\section{Lacosamide}

Indications: Lacosamide is licensed as monotherapy and adjunctive therapy in the treatment of partial-onset seizures with or without secondary generalization in adults, adolescents and children from 4 years of age with epilepsy. Non-licensed use includes the treatment of nonconvulsive and convulsive status epilepticus, neuropathic pain and Restless legs syndrome.

Pharmacokinetics: The pharmacokinetics of lacosamide are linear. The drug is rapidly absorbed after oral ingestion with a Tmax of 1-2 h, a Vd of 0.6-0.7 L/kg and bioavailability of $100 \% .{ }^{90}$ Binding to plasma proteins is $14 \% .{ }^{61}$ Lacosamide is metabolized in the liver by demethylation, primarily by CYP2C19, but CYP3A4 and CYP2C9 may also be involved, to form O-desmethyl lacosamide (30\%) together with other unidentified metabolites (30\%). ${ }^{91}$ The $t \frac{1}{2}$ of lacosamide in adults is $12-14 h{ }^{.91,92}$

Drug-drug pharmacokinetic interactions: Lacosamide is associated with only a few drugdrug pharmacokinetic interactions. Thus the enzyme-inducing AEDs, carbamazepine, phenytoin and phenobarbital enhance elimination of lacosamide and decrease plasma lacosamide concentrations. ${ }^{93-95}$

Lacosamide TDM: The current reference range for lacosamide in plasma is $10-20 \mathrm{mg} / \mathrm{L}$. Lacosamide distributes into saliva and concentrations reflect the non-protein bound concentration in plasma; thus, saliva can be a useful alternative matrix for lacosamide TDM. ${ }^{2,96}$

\section{Lamotrigine}

Indications: Lamotrigine is licensed for the monotherapy treatment of partial seizures and primary and secondarily generalized tonic-clonic seizures in adults and children over 12 years of age and as adjunctive treatment in adults and children over 2 years of age; also for the adjunctive treatment of seizures associated with the Lennox-Gastaut syndrome in adults and children over 2 years of age. Lamotrigine is also licensed for the treatment of bipolar I disorder. Non-licensed uses include treatment of absence seizures, bipolar depression, cluster headaches, migraine, neuropathic pain, peripheral neuropathy, psychosis, schizophrenia and trigeminal neuralgia. 
Pharmacokinetics: The pharmacokinetics of lamotrigine are linear. Following oral ingestion lamotrigine is rapidly absorbed with a Tmax of $1-3 \mathrm{~h}$ and bioavailability of $>95 \% .{ }^{97} \mathrm{It}$ is $66 \%$ bound to plasma proteins and its $\mathrm{Vd}$ is $0.9-1.3 \mathrm{~L} / \mathrm{kg} .{ }^{61}$ Lamotrigine is extensively metabolized in the liver, primarily via UGT1A4, but UGT1A1 and UGT2B7 also contribute, to form 2-N (76\%) and 5-N glucuronides (10\%). ${ }^{97}$ The $\mathrm{t} 1 / 2$ of lamotrigine in adults is $15-35 \mathrm{~h}$. Drug-drug pharmacokinetic interactions: Lamotrigine glucuronidation mainly via UGT1A4 is particularly susceptible to interference and thus there are many drugs that can inhibit or induce the metabolism of lamotrigine. The AEDs that inhibit the metabolism of lamotrigine and therefore increase blood lamotrigine concentrations include: felbamate and valproic acid, the latter AED being particularly potent. In contrast, carbamazepine, eslicarbazepine acetate, methsuximide, oxcarbazepine, phenobarbital, phenytoin, primidone, retigabine and rufinamide induce lamotrigine metabolism and decrease lamotrigine concentrations. ${ }^{70}$ Lamotrigine metabolism can also be affected by various non-epilepsy drugs with aripiprazole, isoniazid and sertraline inhibiting lamotrigine metabolism to cause increased blood concentrations while drugs that induce lamotrigine metabolism and decrease lamotrigine blood concentrations include: acetaminophen, atazanavir, ethambutol, olanzapine, oral contraceptives (estrogen component), rifampicin and ritonavir. 70 Lamotrigine TDM: There are large differences between individuals in the dose to plasma concentration relationship for lamotrigine which can be attributed in part to general pharmacokinetic variability and also by the fact that it is susceptible to many drug-drug pharmacokinetic interactions. Lamotrigine is also associated with potentially life-threatening cutaneous rash which is directly related to the rate of lamotrigine exposure and can be exacerbated by valproic acid which potently inhibits its metabolism. These characteristics make lamotrigine TDM particularly valuable. ${ }^{98}$ The current reference range for lamotrigine in plasma is $2.5-15 \mathrm{mg} / \mathrm{L} .{ }^{1}$ The drug is secreted into saliva where concentrations reflect non-protein bound concentration in plasma; thus saliva can be used as an alternative matrix for lamotrigine TDM. ${ }^{2}$

\section{Levetiracetam}

Indications: Levetiracetam is licensed for monotherapy treatment of partial seizures with or without secondary generalization in patients aged 16 years and older, also for adjunctive 
treatment in adults and children from 1 month of age. In addition, the drug is approved for adjunctive treatment of primary generalized tonic-clonic seizures associated with idiopathic generalized epilepsy and myoclonic seizures in adults and adolescents from 12 years of age with juvenile myoclonic epilepsy. Non-licensed uses include the treatment of absence and myoclonic seizures, the management of benign rolandic epilepsy, childhood occipital epilepsy, progressive myoclonic epilepsy (Unverricht-Lundborg syndrome), severe myoclonic epilepsy in infancy (Dravet syndrome) and status epilepticus.

Pharmacokinetics: The pharmacokinetics of levetiracetam are linear. Following oral ingestion levetiracetam is rapidly absorbed with a Tmax of 1-2 $\mathrm{h}$ and a bioavailability of $>95 \%$. It is $3 \%$ protein bound and its $\mathrm{Vd}$ is $0.5-0.7 \mathrm{~L} / \mathrm{kg} .{ }^{99,61}$ Approximately $30 \%$ of a levetiracetam dose is hydrolyzed, via a type-B esterase enzyme located in whole blood, to produce the metabolite 2-pyrrolidone-N-butyric acid, which is excreted unchanged via the kidneys. ${ }^{100}$ The drug also undergoes a small amount of hepatic metabolism to form two ring hydroxylated metabolites. The remaining $70 \%$ is excreted unchanged via the kidneys. The $\mathrm{t} t / 2$ of levetiracetam in adults, children and the elderly is $6-8 \mathrm{~h}, 5-6 \mathrm{~h}$ and $10-11 \mathrm{~h}$ respectively. ${ }^{99}$

Drug-drug pharmacokinetic interactions: Levetiracetam is subject to few drug-drug pharmacokinetic interactions. However, carbamazepine, lamotrigine, methsuximide, oxcarbazepine, phenobarbital and phenytoin enhance its metabolism resulting in a 20-30\% reduction in plasma levetiracetam concentrations. ${ }^{70}$

Levetiracetam TDM: The current reference range for levetiracetam in plasma is $12-46$ $\mathrm{mg} / \mathrm{L} .^{1,101}$ Levetiracetam is secreted into saliva and concentrations are significantly correlated with those in plasma; thus, saliva may be used as an alternative matrix for levetiracetam TDM. ${ }^{2}$

\section{Oxcarbazepine}

Indications: Oxcarbazepine is licensed for both monotherapy and adjunctive treatment of partial seizures with or without secondary generalization in patients $\geq 6$ years of age. Nonlicensed uses include the treatment of bipolar disorder and trigeminal neuralgia.

Pharmacokinetics: Oxcarbazepine is a pro-drug for the pharmacologically active 10hydroxycarbazepine; which exhibits linear pharmacokinetics. After oral ingestion 
oxcarbazepine is rapidly absorbed so that Tmax for 10 -hydroxycarbazepine is $3-6 \mathrm{~h}$. Its bioavailability is $100 \%$ and its $\mathrm{Vd}$ is $0.75 \mathrm{~L} / \mathrm{kg}$. Plasma protein binding of 10 hydroxycarbazepine is $40 \% .{ }^{102,61}$ Oxcarbazepine is rapidly metabolized, by cytosolic arylketone reductase, to racemic 10-hydroxycarbazepine (also known as S-licabazepine and monohydroxy derivative). The conversion of oxcarbazepine to 10-hydroxycarbazepine is stereoselective and concentrations of the S-enantiomer (which has slightly greater pharmacological activity) are a little higher than those of the R-enantiomer. ${ }^{103,104} 10$ hydroxycarbazepine is metabolized primarily by glucuronidation (51\%) and it also undergoes hydroxylation via CYP isoenzymes to form a dihydrodiol metabolite (28\%). The $\mathrm{t} t / 2$ of 10 -hydroxycarbazepine in adults is $8-15 \mathrm{~h}$.

Drug-drug pharmacokinetic interactions: Oxcarbazepine (10-hydroxycarbazepine) is subject to few drug-drug pharmacokinetic interactions. However, the AEDs carbamazepine, lacosamide, phenobarbital and phenytoin enhance its metabolism resulting in a 15-35\% reduction in plasma 10-hydroxycarbazepine concentrations while verapamil can also decrease plasma 10-hydroxycarbazepine concentrations by $20 \%$ and viloxazine can increase concentrations by $11 \%{ }^{70}$

Oxcarbazepine TDM: Although oxcarbazepine per se is pharmacologically active, the fact that it is rapidly metabolized to 10-hydroxycarbazepine resulting in very low, and often not detectable, blood concentrations, it is now routine practice to monitor only 10hydroxycarbazepine concentrations. Because of large differences between individuals in the oxcarbazepine dose to plasma 10-hydroxycarbazepine concentration relationship, TDM is a valuable adjunct in individualizing treatment. The current reference range for 10hydroxycarbazepine in plasma is $3-35 \mathrm{mg} / \mathrm{L.}^{1} 10$-hydroxycarbazepine is secreted into saliva and there is good correlation with plasma concentrations; thus, saliva can be a used as an alternative matrix for 10-hydroxycarbazepine TDM. ${ }^{2}$

\section{Perampanel}

Indications: Perampanel is licensed for the adjunctive treatment of partial-onset seizures with or without secondary generalization in patients aged 12 years and older. Also, it is licensed for the adjunctive treatment of primary generalized tonic-clonic seizures in adult 
and adolescent patients from 12 years of age with idiopathic generalized epilepsy. Pharmacokinetics: The pharmacokinetics of perampanel are linear. Following oral ingestion perampanel is rapidly absorbed with a Tmax of $0.5-2.5 \mathrm{~h}$ and its bioavailability is $100 \%$. Perampanel has a Vd of $1.1 \mathrm{~L} / \mathrm{kg}$ and is $98 \%$ bound to plasma proteins. ${ }^{61}$ The drug is extensively metabolized in the liver, primarily via CYP3A4, to various metabolites and the $t_{1}$ in adults is $48 \mathrm{~h} .{ }^{105}$

Drug-drug pharmacokinetic interactions: Perampanel is susceptible to drug-drug pharmacokinetic interactions. Thus, the AEDs carbamazepine, oxcarbazepine, phenytoin and topiramate enhance its metabolism and decrease plasma perampanel concentrations whilst ketoconazole inhibits metabolism and increases plasma concentrations. ${ }^{105}$

Perampanel TDM: In the patients that responded to perampanel in the Phase III clinical trials, plasma concentrations ranged from 180 to $980 \mu \mathrm{g} / \mathrm{L}$. This range represents the current putative reference range for perampanel in plasma. ${ }^{105} \mathrm{It}$ is not known whether perampanel is secreted into saliva.

\section{Phenobarbital}

Indications: Phenobarbital is licensed for the treatment of patients of any age for all forms of epilepsy, except absence seizures, as monotherapy or as adjunctive treatment. Nonlicensed uses include the treatment of acute convulsive episodes and status epilepticus, Lennox-Gastaut syndrome, myoclonic seizures, neonatal seizures and for prophylaxis of febrile seizures.

Pharmacokinetics: The pharmacokinetics of phenobarbital are linear. Following oral ingestion phenobarbital is rapidly absorbed with a Tmax of 2-4 $\mathrm{h}$ and its bioavailability is $>90 \%$. It has a Vd of $0.61 \mathrm{~L} / \mathrm{kg}$ and is $48 \%$ bound to plasma proteins. ${ }^{61}$ Phenobarbital is extensively metabolized in the liver, primarily via CYP2C9 but CYP2C19 and CYP2E1 play a minor role, to form $\mathrm{p}$-hydroxy-phenobarbital and via glucuronidation to form an $\mathrm{N}$ glucoside conjugate. The $t \frac{1}{2}$ of phenobarbital in adults is $70-140 \mathrm{~h}$ whilst in newborn it is $100-200 \mathrm{~h}$ indicating that inter-individual clearance is extremely variable. ${ }^{106,107}$

Drug-drug pharmacokinetic interactions: Phenobarbital is susceptible to drug-drug pharmacokinetic interactions, particularly by other AEDs, and most interactions relate to metabolic inhibition. Thus, the AEDs acetazolamide, felbamate, methsuximide, 
oxcarbazepine, phenytoin, retigabine, rufinamide, stiripentol, sulthiame and valproic acid inhibit the metabolism of phenobarbital and increase phenobarbital plasma concentrations; ${ }^{69}$ likewise, chloramphenicol and propoxyphene can also increase plasma concentrations whilst dicoumarol, thioridazone and troleandomycin can decrease plasma phenobarbital concentrations. ${ }^{70}$

Phenobarbital TDM: Because substantial variability in clearance occurs between individuals there are large differences in the dose to plasma concentration relationship. The current reference range for phenobarbital in plasma is $10-40 \mathrm{mg} / \mathrm{L} .{ }^{1}$ Phenobarbital distributes into saliva and salivary phenobarbital concentrations are significantly correlated with both plasma total and free phenobarbital concentrations thus saliva is a useful alternative matrix for phenobarbital TDM. ${ }^{2}$

\section{Phenytoin}

Indications: Phenytoin is licensed for monotherapy and adjunctive therapy of tonic-clonic seizures and focal seizures in patients of any age. It is also approved for treating seizures which occur during or following neurosurgery and/or severe head injury. In addition, phenytoin is licensed for the management of established status epilepticus and for use as monotherapy for the treatment of trigeminal neuralgia. A non-licensed use of phenytoin is for the treatment of cardiac arrhythmias.

Pharmacokinetics: The pharmacokinetics of phenytoin are non-linear due to its saturable metabolism so that clearance decreases with increasing dose. ${ }^{108}$ Very importantly the nonlinearity becomes apparent at different concentrations because the Km or EC50 differs between individual patients. Furthermore, there are many formulations of phenytoin and because of its physicochemical characteristics its pharmacokinetics exhibit significant variability. Thus, absorption of phenytoin following oral ingestion is variable and formulation dependent with a Tmax of 1-12 h. ${ }^{109} \mathrm{Its} \mathrm{Vd}$ is $0.5-0.8 \mathrm{~L} / \mathrm{kg}$ with a bioavailability of $\leq 80 \%$ (which is also formulation dependent) and is $92 \%$ bound to plasma proteins. ${ }^{61}$ Phenytoin is extensively metabolized in the liver, via CYP2C9 (80\%) and CYP2C19 (20\%), to form a p-hydroxy metabolite and a dihydrodiol derivative. The $\mathrm{t} t / 2$ of phenytoin in adults is $30-100 \mathrm{~h}$.

Drug-drug pharmacokinetic interactions: Because phenytoin is associated with saturable 
metabolism which is also readily inhibited and induced, it is subject to more drug-drug pharmacokinetic interactions than any other AED. The AEDs that inhibit phenytoin metabolism and thus increase phenytoin blood concentrations include: acetazolamide, clobazam, eslicarbazepine acetate, felbamate, methsuximide, oxcarbazepine, rufinamide, stiripentol, sulthiame and topiramate. ${ }^{70}$ The effect of carbamazepine, phenobarbital and valproic acid on phenytoin concentrations is conflicting with plasma concentrations decreasing, increasing or remaining the same. ${ }^{70}$

Phenytoin metabolism can also be affected by many non-epilepsy drugs. The drugs that inhibit phenytoin metabolism and increase blood concentrations include: allopurinol, amiodarone, azapropazone, capecitabine, chloramphenicol, chlorphenamine, cimetidine, clarithromycin, clinafloxacin, co-trimoxazole, dicoumarol, diltiazem, disulfiram, doxifluridine, efavirenz, fenyramidol, fluconazole, 5-fluorouracil, fluoxetine, fluvoxamine, imipramine, isoniazid, itraconazole, methaqualone, miconazole, nifedipine, nortriptyline, omeprazole, pasoconazole, propoxyphene, risperidone, ritonavir, sulfadiazine, sulfaphenazole, sulfamethizole, sulfamethoxazole, sulfinpyrazone, tacrolimus, tamoxifen, ticlopidine, trazodone, verapamil, viloxazine and voriconazole. ${ }^{70}$ The drugs that induce phenytoin metabolism and decrease blood concentrations include: carboplatin, cisplatin, diazoxide, loxapine, nelfinavir, nevirapine, rifampicin and theophylline. ${ }^{70}$

Phenytoin TDM: Not only does phenytoin exhibit non-linear pharmacokinetics, but nonlinearity occurs at different doses for different patients and therefore it is impossible to anticipate the blood concentration, and therapeutic response, that will occur with a specific patient. Additionally, phenytoin is susceptible to many drug-drug pharmacokinetic interactions so that overall there are large differences between individuals in the dose to plasma concentration relationship and in phenytoin elimination. Phenytoin TDM is therefore invaluable for guiding treatment and the current reference range in plasma is $10-20 \mathrm{mg} / \mathrm{L.}{ }^{1}$ Phenytoin distributes into saliva with salivary concentration being similar to the non-protein bound concentration in plasma and is thus a useful alternative matrix for phenytoin TDM. ${ }^{2}$

\section{Piracetam}

Indications: Piracetam is licensed for the adjunctive treatment of myoclonus of cortical origin irrespective of etiology. 
Pharmacokinetics: The pharmacokinetics of piracetam are linear. Following oral ingestion piracetam is rapidly absorbed with a Tmax of $0.5-1.5 \mathrm{~h}$ and a bioavailability of $100 \%$. Its $\mathrm{Vd}$ is $0.6 \mathrm{~L} / \mathrm{k}$ and is not bound to plasma proteins. Piracetam is not metabolized being cleared entirely by renal elimination with a $t \frac{1}{2}$ of $4-6 \mathrm{~h}$ in adults with normal renal function. ${ }^{110}$ Drug-drug pharmacokinetic interactions: To-date there have been no drug-drug pharmacokinetic interactions associated with piracetam. ${ }^{70}$

Piracetam TDM: There are no data relating plasma concentrations of piracetam with that of seizure suppression or adverse effects. Clearly, however, measurement of piracetam plasma concentrations can be helpful for ascertaining adherence, investigating malabsorption issues and in cases of suspected toxicity. It is not known whether piracetam is secreted into saliva.

\section{Pregabalin}

Indications: Pregabalin is licensed for the adjunctive treatment of partial seizures with or without secondary generalization in adults. It is also approved for the treatment of generalized anxiety disorders and for peripheral and central neuropathic pain. Nonlicensed uses include the treatment of panic disorder and social anxiety disorder. Pharmacokinetics: The pharmacokinetics of pregabalin are linear. Following oral ingestion pregabalin is rapidly absorbed with a Tmax of $1-2 \mathrm{~h}$, bioavailability is $>90 \%$ and its $\mathrm{Vd}$ is $0.57 \mathrm{~L} / \mathrm{kg}$. Pregabalin is not bound to plasma proteins nor is it metabolized, being cleared entirely by renal elimination with a $\mathrm{t} \frac{1}{2}$ of $5-7 \mathrm{~h}$ in adults having normal renal function. ${ }^{111,61}$ Drug-drug pharmacokinetic interactions: There are only two drug-drug pharmacokinetic interactions reported with pregabalin namely gabapentin and phenytoin can decrease pregabalin plasma concentrations by $18-30 \% .^{70}$

Pregabalin TDM: Little information is available regarding therapeutic plasma concentrations of pregabalin. However, in samples collected at random times relative to dose from clinical trials where patients were maintained on $600 \mathrm{mg} /$ day, plasma pregabalin concentrations ranged 0.9-14.2 $\mathrm{mg} / \mathrm{L}$. The current reference range for pregabalin in plasma is $2-8 \mathrm{mg} / \mathrm{L}$. It is not known whether pregabalin is secreted into saliva. ${ }^{2}$

\section{Primidone}


Indications: Primidone is licensed for both monotherapy and polytherapy of generalized tonic-clonic seizures and psychomotor and focal seizures in adults and children. It is also approved for the management of Jacksonian seizures, myoclonic jerks and akinetic attacks and for the treatment of essential tremor. Non-licensed uses include the treatment of juvenile myoclonic epilepsy and of primarily generalized tonic-clonic seizures.

Pharmacokinetics: The pharmacokinetics of primidone are linear. Following oral ingestion primidone is rapidly absorbed with a Tmax of $2-4 \mathrm{~h}$ and a bioavailability of $>90 \%,{ }^{112} \mathrm{Vd}$ is $0.5-0.8 \mathrm{~L} / \mathrm{kg}$ and it is $33 \%$ bound to plasma proteins. ${ }^{61}$ Primidone is extensively metabolized in the liver to produce two major pharmacologically active metabolites, phenobarbital and phenyl-ethyl-malondiamide. Both metabolites accumulate in plasma and are responsible for much of the drugs action. The $t \frac{1 / 2}{2}$ of primidone in adults is $7-22 \mathrm{~h}$, whilst in children it is $5-11 \mathrm{~h}$ and in newborns it is $8-80 \mathrm{~h} .{ }^{10}$

Drug-drug pharmacokinetic interactions: The metabolism of primidone can be both induced and inhibited, but there are only a few drug-drug pharmacokinetic interactions involving primidone. The AEDs that decrease plasma primidone concentrations include: acetazolamide, carbamazepine and phenytoin, whilst clobazam, ethosuximide and stiripentol increase concentrations. Interestingly, whilst methsuximide, sulthiame and valproic acid do not affect plasma primidone concentrations, they increase plasma phenobarbital concentrations; furthermore, isoniazid and nicotinamide can increase primidone blood concentrations. ${ }^{70}$ Finally, as primidone is metabolized to phenobarbital, all the interactions highlighted earlier for phenobarbital will also apply to primidone. Primidone TDM: During therapy with primidone it is common practice to monitor only phenobarbital since adverse effects from a high plasma phenobarbital concentration is more likely to limit a primidone dosage increase. However, it can be useful to determine both primidone and phenobarbital on occasion (e.g. ascertaining compliance and identifying and characterizing drug-drug pharmacokinetic interactions). The current reference range for primidone in plasma is $5-10 \mathrm{mg} / \mathrm{L} .{ }^{1}$ Primidone is secreted into saliva and total plasma concentrations are significantly correlated with those in saliva. Thus, saliva can be used as an alternative matrix for primidone TDM. ${ }^{2}$

\section{Rufinamide}


Indications: Rufinamide is licensed for the adjunctive treatment of seizures associated with the Lennox-Gastaut syndrome in patients 4 years and older. Non-licensed use includes the treatment of partial seizures, epileptic spasms, myoclonic-astatic epilepsy and status epilepticus.

Pharmacokinetics: The pharmacokinetics of rufinamide are linear up to a dose of about $1600 \mathrm{mg} /$ day and thereafter it is nonlinear. Following oral ingestion rufinamide is absorbed with a Tmax of 4-6 h. Food increases absorption and bioavailability decreases with increasing dose. ${ }^{113}$ Its $\mathrm{Vd}$ is $0.71-1.14 \mathrm{~L} / \mathrm{kg}$ and rufinamide is $28 \%$ bound to plasma proteins. ${ }^{114,61}$ The drug is extensively metabolized in the liver, primarily by hydrolysis, to form a carboxylic acid derivative which in turn is glucuronidated and the $t \frac{1}{2}$ of rufinamide in adults is $6-10$ h. ${ }^{114}$

Drug-drug pharmacokinetic interactions: Rufinamide is subject to some drug-drug pharmacokinetic interactions. Its metabolism can be both induced and inhibited with carbamazepine, methsuximide, oxcarbazepine, phenobarbital, phenytoin, primidone and vigabatrin enhancing its elimination to decrease plasma rufinamide concentrations whilst valproic acid inhibits its elimination and increases plasma concentrations. ${ }^{70,89}$

Rufinamide TDM: That rufinamide is associated with non-linear pharmacokinetics resulting in large differences between individuals in the dose to plasma concentration relationship makes rufinamide an excellent candidate for TDM. The reference range for rufinamide in plasma in patients with Lennox-Gastaut syndrome is $30-40 \mathrm{mg} / \mathrm{L}$, however, lower concentrations may prove effective in other seizure types. ${ }^{114,115}$ Rufinamide distributes into saliva and reflects the non-protein bound drug concentrations in plasma. Thus, saliva can be used as an alternative matrix for rufinamide TDM. ${ }^{2}$

\section{Stiripentol}

Indications: Stiripentol is licensed for the adjunctive treatment of seizures in children with severe myoclonic epilepsy in infancy (Dravet Syndrome). Non-licensed use includes the adjunctive treatment with carbamazepine in children with refractory partial seizures.

Pharmacokinetics: The pharmacokinetics of stiripentol are non-linear due to saturarble metabolism so that clearance decreases with increasing dose. ${ }^{116}$ Following oral ingestion, stiripentol is rapidly absorbed with a Tmax of $0.5-2.0 \mathrm{~h}$, however, its bioavailability has yet 
to be determined. ${ }^{117}$ Its $\mathrm{Vd}$ has not been determined but stiripentol is $96 \%$ bound to plasma proteins. ${ }^{61}$ Stiripentol is extensively metabolized in the liver, primarily by demethylation and glucuronidation, to 13 metabolites. ${ }^{118,119}$ The $t \frac{1}{2}$ of stiripentol is dose dependent but generally in the range $4.5-13 \mathrm{~h}$.

Drug-drug pharmacokinetic interactions: Stiripentol it subject to some drug-drug pharmacokinetic interactions. The AEDs carbamazepine, phenobarbital, phenytoin and primidone enhance its elimination and decreases stiripentol plasma concentrations whilst clobazam decreases its elimination and increases plasma concentrations. ${ }^{70}$

Stiripentol TDM: That stiripentol is associated with both non-linear pharmacokinetics and drug-drug pharmacokinetic interactions which results in large differences between individuals in the dose to plasma concentration relationship. These factors make stiripentol an excellent candidate for TDM. The reference range for stiripentol in plasma of Dravet syndrome patients is $8-12 \mathrm{mg} / \mathrm{L} .{ }^{120}$ For controlling absence seizures in children the reference range is $4-22 \mathrm{mg} / \mathrm{L}^{121}$ It is not known whether stiripentol is secreted into saliva. ${ }^{2}$

\section{Sulthiame}

Indications: Sulthiame is licensed for the treatment of rolandic epilepsy.

Pharmacokinetics: The pharmacokinetics of sulthiame are linear. Following oral ingestion sulthiame is rapidly absorbed with a Tmax of $1.0-5.0 \mathrm{~h}$ and a bioavailability of $100 \%$. Its $\mathrm{Vd}$ has not been determined and is $29 \%$ bound to plasma proteins. Sulthiame undergoes moderate metabolism in the liver via unknown isoenzymes to unknown metabolites. It undergoes renal elimination (80-90\%) with half-life values in children of 5-7 h. ${ }^{122}$ Drug-drug pharmacokinetic interactions: To-date only a few drug-drug pharmacokinetic interactions have been associated with sulthiame. Carbamazepine and primidone can increase the elimination of sulthiame and decrease sulthiame plasma concentrations. Also, antacids containing magnesium trisilicate, bismuth oxycarbonate and magnesium oxide can decrease the gastrointestinal absorption of sulthiame and decrease plasma sulthiame concentrations. ${ }^{10}$

Sulthiame TDM: The current reference ranges for sulthiame in plasma in children and adults on polytherapy AEDs is $2-10 \mathrm{mg} / \mathrm{L}$ and $1-3 \mathrm{mg} / \mathrm{L}$ respectively. It is not known whether sulthiame is secreted into saliva. 


\section{Tiagabine}

Indications: Tiagabine is approved for the adjunctive treatment of partial seizures with or without secondary generalization in adults and children aged 12 years and above. Nonlicensed use includes the treatment of anxiety disorders and neuropathic/chronic pain. Pharmacokinetics: The pharmacokinetics of tiagabine are linear. After oral ingestion tiagabine is rapidly absorbed with a Tmax of $0.5-2 \mathrm{~h}$ and a bioavailability of $\leq 90 \%$. Its $\mathrm{Vd}$ is $1.0 \mathrm{~L} / \mathrm{kg}$ and is $98 \%$ bound to plasma proteins. ${ }^{61}$ Tiagabine is extensively metabolized in the liver, primarily via CYP3A4, to form two 5-oxo-tiagabine isomers (60\%) and various other unidentified metabolites $(40 \%) .{ }^{123}$ The $\mathrm{t}_{1 / 2}$ of tiagabine is $5-9 \mathrm{~h}$.

Drug-drug pharmacokinetic interactions: Tiagabine is subject to only a few drug-drug pharmacokinetic interactions. The AEDs carbamazepine, phenobarbital, phenytoin, pregabalin and primidone enhance its elimination and decrease plasma tiagabine concentrations. In contrast, cimetidine and gemfibrozil decrease tiagabine elimination and increase plasma concentrations. ${ }^{70}$ Valproic acid can displace tiagabine from plasma protein binding sites, increasing the free fraction of tiagabine. ${ }^{124}$

Tiagabine TDM: The current reference range for tiagabine in plasma is $0.02-0.2 \mathrm{mg} / \mathrm{L}$. However, Uthman et al 1998 observed that for their patient group with complex partial seizures, a trough concentration above $0.04 \mathrm{mg} / \mathrm{L}$ was associated with good seizure control whilst concentrations of $0.4 \mathrm{mg} / \mathrm{L}$ were associated with CNS toxicity. ${ }^{125}$ It is not known whether tiagabine is secreted into saliva. ${ }^{2}$

\section{Topiramate}

Indications: Topiramate is licensed for monotherapy treatment in adults and children aged 6 years and above who have generalized tonic-clonic seizures and partial seizures with or without secondarily generalization; also for adjunctive therapy for adults and children aged 2 years and above. It is also approved as adjunctive treatment for seizures associated with Lennox-Gastaut syndrome and primary generalized tonic-clonic seizures. In addition topiramate is approved for the treatment of migraine. Non-licensed use includes the treatment of a variety of seizure disorders (e.g. absence seizures, infantile spasms [West syndrome], juvenile myoclonic epilepsy, myoclonic astatic epilepsy [Doose syndrome], 
progressive myoclonic epilepsy and severe myoclonic epilepsy in infancy [Dravet syndrome]) and non-epilepsy conditions (e.g. bipolar disorder, binge-eating disorder, cluster headaches and neuropathic pain).

Pharmacokinetics: The pharmacokinetics of topiramate are linear. After oral ingestion topiramate is rapidly absorbed with a Tmax of $2-4 \mathrm{~h}$ and a bioavailability of $>80 \%$. Its $\mathrm{Vd}$ is 0.6-0.8 L/kg and it is $20 \%$ bound to plasma proteins. ${ }^{126,61}$ Approximately $50 \%$ of the dose undergoes hepatic metabolism via CYP isoenzymes which have not been identified. The $t \frac{1}{2}$ of topiramate in adults is $20-30$ h. ${ }^{127,128}$

Drug-drug pharmacokinetic interactions: Topiramate is subject to various drug-drug pharmacokinetic interactions with carbamazepine, eslicarbazepine acetate, methsuximide, oxcarbazepine, phenobarbital, phenytoin, primidone and valproic acid enhancing its elimination to decrease topiramate plasma concentrations and several non-epilepsy drugs including diltiazem, hydrochlorothiazide, lithium, metformin, propranolol, posaconazole and sumatriptan decreasing topiramate elimination and increasing plasma concentrations. ${ }^{70}$ Topiramate TDM: The current reference range for topiramate in plasma is $5-20 \mathrm{mg} / \mathrm{L} .{ }^{1}$ Topiramate is secreted into saliva and total plasma concentrations are significantly correlated with those in saliva. Thus, saliva can be used as an alternative matrix for topiramate TDM. ${ }^{2}$

\section{Valproate}

Indications: Valproate is licensed for monotherapy or adjunctive treatment of any form of epilepsy in patients of any age. Non-licensed use includes the treatment of a variety of seizure disorders (e.g. febrile seizures, infantile spasms [West syndrome], juvenile myoclonic epilepsy, Lennox-Gastaut syndrome, neonatal seizures and refractory status epilepticus) and non-epilepsy conditions (e.g. bipolar depression, psychosis and schizophrenia).

Pharmacokinetics: The pharmacokinetics of valproic acid are non-linear because it exhibits saturable binding to plasma proteins. ${ }^{129}$ Valproic acid is rapidly absorbed after oral ingestion; however, Tmax is formulation dependent and variable (1-7 hours). Bioavailability is generally $>90 \%$ but may be only $70 \%$ for extended release preparations. Plasma protein binding is concentration dependent and can vary from $74 \%$ to $93 \% .{ }^{61}$ Its $\mathrm{Vd}$ is $0.13-0.19$ 
$\mathrm{L} / \mathrm{kg}$. Valproic acid undergoes extensive and complex hepatic metabolism, via a number of CYP and UGT isoenzymes, with more than 25 metabolites being identified; some of which are pharmacologically active. The $t / 2$ of valproic acid in adults is $12-16 \mathrm{~h}$ and in children it is $8-13$ h. ${ }^{130}$

Drug-drug pharmacokinetic interactions: The metabolism of valproic acid is readily induced or inhibited and thus it is susceptible to many drug-drug pharmacokinetic interactions. The AEDs carbamazepine, eslicarbazepine acetate, ethosuximide, lamotrigine, methsuximide, phenobarbital, phenytoin, primidone, tiagabine and topiramate enhance the elimination of valproic acid and decrease plasma concentrations. Plasma valproic acid concentrations are also decreased by several non-epilepsy drugs including amikacin, cisplatin, diflunisal, doripenem, efavirenz, ertapenem, imipenem, meropenem, methotrexate, naproxen, oral contraceptives, panipenam, rifampicin and ritonavir. ${ }^{70}$ In contrast, the AEDs clobazam, felbamate and stiripentol and the non-epilepsy drugs bupropion, chlorpromazine, cimetidine, erythromycin, guanfacine, isoniazid, lithium, sertraline and verapamil inhibit the metabolism of valproic acid and increase plasma concentrations. ${ }^{70}$

Valproic acid TDM: That valproic acid is associated with non-linear pharmacokinetics, due to saturable plasma protein binding, resulting in large differences between individuals in the dose to plasma concentration relationship makes it a good candidate for TDM and the current reference range for valproic acid in plasma is $50-100 \mathrm{mg} / \mathrm{L} .{ }^{1}$ However, measurement of non-protein bound concentration may be more useful clinically because of the large variability in protein binding. Because distribution of valproic acid into saliva is reported to be erratic, saliva is not a useful alternative matrix for valproic acid TDM; ${ }^{2}$ however, a recent publication has suggested that valproic acid can be monitored in saliva and that the concentrations reflect the non-protein bound concentration. ${ }^{131}$ These somewhat contradictory data require further validation.

\section{Vigabatrin}

Indications: Vigabatrin is approved for the adjunctive treatment of partial seizures with and without secondary generalization and also for monotherapy treatment of infantile spasms (West Syndrome).

Pharmacokinetics: The pharmacokinetics of vigabatrin are linear. After oral ingestion 
vigabatrin is rapidly absorbed with a Tmax of $1-2 \mathrm{~h}$. Bioavailability is $60-80 \%$, Vd is $0.8 \mathrm{~L} / \mathrm{kg}$ and the drug is $17 \%$ bound to plasma proteins. ${ }^{61}$ Vigabatrin is not metabolized and is cleared entirely by renal elimination with a $t_{1} / 2$ in adults of $5-8 \mathrm{~h} .{ }^{132}$

Drug-drug pharmacokinetic interactions: With the exception of felbamate which increases its elimination, vigabatrin is not the subject of drug-drug pharmacokinetic interactions. ${ }^{70}$ Vigabatrin TDM: TDM of vigabatrin is generally considered not to be very helpful because of its mechanism of action, whereby it increases brain $y$-amino butyric acid (GABA) concentrations by selectively and irreversibly binding to GABA-transaminase (GABA-T). ${ }^{133}$ Since GABA metabolism depends upon re-synthesis of GABA-T, GABA concentrations remain elevated for some time after vigabatrin has been eliminated from plasma. ${ }^{134}$ However, TDM may be useful in patients with renal impairment, also to ascertain compliance, where malabsorption is suspected and in cases of suspected overdose. The current reference range for vigabatrin in plasma is $0.8-36 \mathrm{mg} / \mathrm{L} .{ }^{1}$ Vigabatrin is transported into saliva but there are no data regarding the correlation between salivary and plasma vigabatrin concentrations; therefore, it is currently uncertain whether saliva could be used as an alternative matrix for vigabatrin TDM. ${ }^{2}$

\section{Zonisamide}

Indications: Zonisamide is licensed for the monotherapy treatment of partial seizures, with or without secondary generalization in adults with newly diagnosed epilepsy. It is also licensed for the adjunctive treatment of partial seizures with and without secondary generalization in adults, adolescents and children aged 6 years and above. Non-licensed use includes the treatment of absence seizures, infantile spasms (West syndrome), juvenile myoclonic epilepsy, Lennox-Gastaut syndrome, myoclonic astatic epilepsy (Doose syndrome) myoclonic seizures and progressive myoclonic epilepsy (Unverricht-Lundborg and Lafora disease). Zonisamide is also prescribed for the treatment of binge-eating disorder, bipolar disorder, migraine and neuropathic pain.

Pharmacokinetics: The pharmacokinetics of zonisamide are linear. The drug is rapidly absorbed after oral ingestion with a Tmax of $2-5 \mathrm{~h}$ and bioavailability of $>90 \%$. It is $40 \%$ bound to plasma proteins ${ }^{61}$ and also binds to erythrocytes via a high affinity, low capacity binding site; ${ }^{135} \mathrm{Vd}$ is $1.0-1.9 \mathrm{~L} / \mathrm{kg}$. Zonisamide is extensively metabolized in the liver, 
primarily by acetylation, to form $\mathrm{N}$-acetyl zonisamide and reduction via CYP3A4 to form 2sulfamoylacetylphenol which is subsequently glucuronidated. ${ }^{136}$ The $t \frac{1}{2}$ of zonisamide in adults is $50-70$ h. ${ }^{137,138}$

Drug-drug pharmacokinetic interactions: Zonisamide is associated with only a few drugdrug pharmacokinetic interactions and these entail induction of metabolism. Thus carbamazepine, phenobarbital, phenytoin, primidone and risperidone enhance the elimination of zonisamide and decrease plasma concentrations. ${ }^{70}$

Zonisamide TDM: The current reference range for zonisamide in plasma is $10-40 \mathrm{mg} / \mathrm{L} .{ }^{1,139}$ Zonisamide distributes into saliva, but there is limited information regarding salivary concentrations compared with those in plasma. $^{2}$

\section{Sample types}

AED TDM is normally undertaken in either plasma or serum and concentrations in each are similar. Other sample types include dried blood spots and saliva. Ideally AED TDM should entail the measurement of the "free" (non-protein bound) drug because it is that concentration in plasma which reflects the concentration in the brain and therefore the therapeutic outcome. However, in most clinical settings the relationship between the free concentration and the bound is constant; therefore, measurement of total concentrations (free plus bound) is adequate. Furthermore, measurement of total concentration is easier, less time consuming and cheaper. However, in clinical settings where protein binding might be disturbed (e.g. during pregnancy, post-surgery or during hepatic or renal disease) clinical management would be best guided by measurement of free concentrations, particularly for highly protein bound AEDs such as phenytoin, carbamazepine and valproate. In the case of protein binding displacement interactions (e.g. the displacement of phenytoin by valproic acid), total plasma phenytoin concentration are misleading because therapeutic and toxic effects are more likely to be observed at total concentrations that are lower than expected.

Saliva AED monitoring is widely practiced in the USA and its use in the UK is increasing. ${ }^{2}$ The advantages are many and include:- 1) concentration reflects the nonprotein bound, pharmacologically active component in plasma; 2) saliva is easier to collect than blood and many patients prefer saliva sampling and;3) the standard 
analytical methods can normally be adapted to accept saliva specimens. However, during the collection of saliva, contamination from unhealthy gums and dental caries can occur and thus there is risk of spurious analytical results. ${ }^{140,141}$ Furthermore, it has been demonstrated that saliva can be contaminated for at least 2 hours after holding a carbamazepine tablet in the mouth for just 5 seconds. ${ }^{142}$ Similarly, phenytoin may persist for up to 3 hours after ingestion. ${ }^{143}$ Also, if the patient is a tablet chewer or is prescribed a chewable or a liquid formulation and has dental caries, then pockets of drug can be deposited, which could contaminate the saliva sample Also, if a liquid preparation is prescribed and saliva collected within a couple of hours of the dose, a false high concentration may result. ${ }^{144}$ Finally, unhealthy, bleeding gums (gingivitis and gingival hyperplasia), which is common in patients prescribed phenytoin) would contaminate the saliva specimen with blood, and it may not be possible to obtain a satisfactory salivary measurement in such cases. Although ideally a predose morning steady-state saliva sample should be collected, a post-dose sample should only be collected after the mouth is thoroughly flushed.

The use of dried blood spots (DBS) is increasing ${ }^{145-151}$. The advantages of dried blood spots include:1) only a fingerpick of blood is required and;2) also when specimen collection is required at a specific time e.g. to ascertain whether transient toxicity is drug related. In addition, for both saliva and dried blood spots a phlebotomist is not required so that the specimen can be collected by a carer at home and posted to the laboratory for analysis in advance of the next clinic visit. A major disadvantage is that analysis of nonprotein-bound concentrations cannot be undertaken. Also, caution is warranted because whilst DBS may be relatively easy to establish an assay for AED TDM in general, it is imperative that a clinical validation study is undertaken to demonstrate acceptable agreement between DBS and liquid samples. ${ }^{152}$

\section{Sample collection and submission}

It is vital to collect an appropriate specimen and submit it to the laboratory together with a request form containing all the required information, such as dose of medication, other 
prescribed drugs, date/time blood was collected and date/time of last dose of medication, to allow proper interpretation of analytical findings. Since a considerable number of AEDs are currently available which have similar adverse (and beneficial) effects; and multidrug therapy is frequently necessary (particularly for refractory patients), it is recommended that the specimen be submitted to a laboratory which can provide complete analytical coverage and undertake a multidrug analysis. In addition, the laboratory should be able to determine non-protein bound drug concentrations.

For blood collection, the type of anticoagulant is important and plasma derived from common types do not necessarily provide similar quantitative AED results. ${ }^{153}$ However, there has been some debate as to whether or not the use of separator gel tubes may result in decreases in serum concentrations of some of the first generation AEDs (e.g. phenytoin, carbamazepine and phenobarbital). Bailey et al. $1988^{154}$ reported no significant change in phenytoin, carbamazepine and phenobarbital concentrations over a week when the collection tubes were stored in the refrigerator. Similarly, Dasgupta et al., $2000^{155}$ reported no effect on phenytoin, phenobarbital and valproic acid concentrations but a small timedependent effect on carbamazepine concentrations was observed. However, at room temperature phenytoin concentrations decrease progressively at 24 hours and 48 hours post sample collection. ${ }^{156}$ Free phenytoin and phenobarbital concentrations may also be affected by gel collection tubes. ${ }^{157,158}$ However, overall it would appear that the use of separator gel tubes may result in decreases in phenytoin, carbamazepine and phenobarbital serum concentrations, particularly if small sample volumes are used or the serum remains in contact with the barrier gel for a prolonged period of time, particularly at room temperature. However, the reductions noted to date have not been large enough to preclude their clinical use. Nevertheless, gel tubes should be used with caution and avoided where possible; if their use is unavoidable the specimen should be stored refrigerated and centrifuged at the earliest and the serum transferred to another plain tube to await analysis. Finally, because gel tubes may disturb protein binding they should be avoided when non-protein bound drug concentrations are being determined. The effect of gel tubes on the serum concentrations of the new second and third generations AEDs is presently under investigation. ${ }^{153}$ Data to date suggest that gel tubes do not have any effect 
that can be considered of clinical significance.

Sampling time is very important. Unless toxicity is suspected, trough concentrations provide the most useful information, and samples should be collected just before the next scheduled dose and preferably in the morning after an overnight fast. Properly timed sampling should be collected at steady-state when drug absorption and distribution are completed. To determine when steady-state will occur, an estimate of the elimination halflife is usually used; ideally the patient's individual clearance (half-life) should be used. Thus, if a drug has a half-life of 12 hours, the clinician should wait 60 hours ( $5 \times 12$ hours) after dose adjustment before determining the plasma concentration. Samples drawn before steady-state is achieved will result in lower than predicted drug concentrations, which may prompt higher than necessary dosage adjustments. For AEDs with long half-lives (e.g. ethosuximide, phenobarbital, phenytoin, perampanel, zonisamide) the fluctuation in plasma drug concentration during a dosing interval is negligible, and samples can be collected at any time, but for the majority of AEDs which have shorter half-lives (e.g. brivaracetam, carbamazepine, levetiracetam, lamotrigine, lacosamide, topiramate) it is important to standardize sampling time in relation to dose.

AEDs are considered to be stable in plasma and saliva under normal postal conditions and can therefore be routinely submitted to the laboratory by normal first class post (ensuring that postal regulations are satisfied). Stability of AEDs in saliva samples has however only been evaluated for carbamazepine, gabapentin, lamotrigine, levetiracetam, oxcarbazepine, phenytoin, phenobarbital, topiramate and zonisamide. ${ }^{159-162}$

\section{Future perspectives}

\section{Population pharmacokinetic modelling}

Because patients exhibit widely varying pharmacokinetic and pharmacodynamic responses, they need careful management. Increasingly it has been highlighted that TDM based only on measurement of plasma concentrations without software for adaptive control of dosage has often been ineffective, even for AEDs with well-established reference ranges. Using population pharmacokinetic modelling, to develop the initial dosage regime 
and followed by TDM measurement, a useful initial dosage regimen can be computed to best achieve the desired target goal for a particular patient. ${ }^{163}$

A significant advantage of the individualized patient model is that it allows dosage adjustments without having to achieve steady-state before measuring plasma concentrations. This is especially important when individual patient's clearance varies significantly from mean half-life values. This approach can help clinicians rapidly establish an effective goal-oriented target, identifying fast and slow metabolizers and evaluating potential pharmacokinetic causes for alteration in drug utilization including drug-drug interactions involving inhibition and induction processes. ${ }^{164}$ With the increasing availability of population pharmacokinetic modelling software, it can be anticipated that more and more AED TDM providers will provide this bespoke patient-oriented service.

\section{Pharmacogenomics}

Increasingly, pharmacogenomics is being used to identify genetic variants which might impact on drug behavior both in terms of its blood distribution/concentration and consequent efficacy and in terms of its toxicity. However, typically, studies have been undertaken to identify variants, most of which have only modest effects on drug concentrations, and rarely seek to confirm the magnitude of the predicted effect in individual patients.

For example, because pharmacogenetic factors may play a role for the pharmacokinetic variability of lamotrigine there has been much recent interest in the UGT1A4*3 variant in specific populations since UGT1A4 is the major enzyme responsible for the metabolism (glucuronidation) of lamotrigine. ${ }^{165-168}$ However, routine genotyping in individual patients and subsequent use of the data to guide clinical management does not occur and such a TDM service could prove invaluable.

Also, AED-related severe cutaneous adverse reactions consequent to genetic variants have been well described for phenytoin, carbamazepine and lamotrigine. ${ }^{169-172}$ Nevertheless, genetic known variants are rarely determined in individual patients and such a TDM service, which will allow dosing according to genotype, could prove invaluable. 


\section{Summary}

AED TDM is now a well-established tool for the management of epilepsy as it allows individualization of treatment consequent to the specific nature of epilepsy and the pharmacokinetic variability of AEDs. While drug measurements are mostly undertaken in plasma many AEDs can be readily monitored in saliva or dried blood spots. For highly protein-bound AEDs, saliva has, in many cases, the advantage of reflecting the free, nonprotein bound, pharmacologically active concentration of drug in plasma. The clinical value of monitoring free drug concentrations is particularly applicable to AEDs such as phenytoin and valproic acid, which are $>90 \%$ protein bound.

Reference ranges have been defined for almost all AEDs and these ranges are useful in that most patients are expected to exhibit optimum clinical response if their plasma concentrations are within these ranges. However, due to individual variation in seizure severity and seizure type, some patients may require concentrations outside the reference ranges. Determination and application of the "individual therapeutic concentration" has many advantages in that it is specific for an individual whereby seizure freedom with good tolerability or optimum seizure control with minimal adverse effects is achieved.

The principal indications for AED TDM are summarized in Table 2 and Table 4 details recommendations for their optimal use.

\section{Acknowledgement and disclosure}

This article was prepared at the invitation of the Reviews Editor of Therapeutic Drug Monitoring

The work undertaken by Professor P N Patsalos was undertaken at UCLH/UCL who received a proportion of funding from the Department of Health's NIHR Biomedical Research Centres funding scheme.

Professor P N Patsalos and Drs D J Berry and E P Spencer have no conflict of interests to declare with regards to this publication. 


\section{References}

1. Patsalos PN, Berry DJ, Bourgeois BFD, et al. Antiepileptic drugs: Best practice guidelines for therapeutic drug monitoring: A position paper by the Subcommission on Therapeutic Drug Monitoring, ILAE Commission on Therapeutic Strategies. Epilepsia. 2008;49:1239-1276.

2. Patsalos PN, Berry DJ. Therapeutic drug monitoring of antiepileptic drugs by use of saliva. Ther Drug Monit. 2013:35:4-29.

3. Johannessen SI, Battino D, Berry DJ, et al. Therapeutic drug monitoring of the newer antiepileptic drugs. Ther Drug Monit. 2003;25:347-263.

4. Woo E, Chan YM, Yu YL, et al. If a well-stabilized epileptic patient has a subtherapeutic antiepileptic drug level, should the dose be increased? A randomized prospective study. Epilepsia. 1988;29:129-139.

5. Johannessen Landmark C, Johannessen SI, Tomson T. Dosing strategies of antiepileptic drugs: from a standard dose for all to individualised treatment by implementation of therapeutic drug monitoring. Epileptic Disord. 2016;18:367-383.

6. Samsonsen C, Reimers A, Bråthen G, et al. Nonadherence to treatment causing acute hospitalizations in people with epilepsy: an observational, prospective study. Epilepsia. 2014;55:e125-e128.

7. Specht U, Elsner H, May TW, et al. Postictal serum levels of antiepileptic drugs for detection of noncompliance. Epilepsy Behav. 2003;4:487-495.

8. Mucklow JC, Dollery CT. Compliance with anticonvulsant therapy in a hospital clinic and community. Br J Clin Pharmacol.1978;6:75-79.

9. Eadie MJ. Indications for plasma drug monitoring in patients with epilepsy. Implications for reducing costs. Pharmacoeconomics. 1997;11:343-349.

10. Patsalos PN, St Louis EK. The epilepsy prescriber's guide to antiepileptic drugs. Cambridge: Cambridge University Press, $3^{\text {rd }}$ Edition, 2018.

11. Aman MG, Paxton JW, Field CJ, et al. Prevalence of toxic anticonvulsant drug concentrations in mentally retarded persons with epilepsy. Am J Ment Defic. 1986;90:643-650. 
12. Djordjevic S, Kilibarda V, Vucinic S, et al. Toxicokinetics and correlation of carbamazepine salivary and serum concentrations in acute poisoning. Vosjnosanit Pregl. 2012;69:389-393.

13. Perucca E. Clinical pharmacokinetics of new generation antiepileptic drugs at the extremes of age. Clin Pharmacokinet. 2006;45:351-363.

14. Hadjiloizou SM, Bourgeois BF. Antiepileptic drug treatment in children. Expert Rev Neurother. 2007;7:179-193.

15. Italiano D, Perucca E. Clinical pharmacokinetics of new-generation antiepileptic drugs at the extremes of age: an update. Clin Pharmacokinet. 2013;52:627-645.

16. Walson PD. Role of therapeutic drug monitoring (TDM) in pediatric anti-convulsant drug dosing. Brain Dev.1994;16:23-26.

17. Lifshitz M, Ben-Zvi Z, Gorodischer R. Monitoring phenytoin therapy using citric acidstimulated saliva in infants and children. Ther Drug Monit. 1990;12:334-338.

18. Mucklow JC, Bacon CJ, Hierons AM, et al. Monitoring of phenobarbitone and phenytoin therapy in small children by salivary samples Ther Drug Monit. 1981;3:275277.

19. Cai WM, Zhu GZ, Chen G. Free phenytoin monitoring in serum and saliva of patients in China. Ther Drug Monit. 1993;15:31-34.

20. de Wildt SN, Kerkvliet KTM, Wazenberg MGA, et al. Use of saliva in therapeutic drug monitoring of caffeine in preterm infants. Ther Drug Monit. $2001 ; 23: 250-254$.

21. Perucca E. Drug metabolism in pregnancy, infancy and childhood. Pharmacol Ther. 1987;34:129-143.

22. Tomson T, Battino D. Pharmacokinetics and therapeutic drug monitoring of newer antiepileptic drugs during pregnancy and puerperium. Clin Pharmacokinet. 2007;46:209-219.

23. Tomson T, Palm R, Kallen K, et al. Pharmacokinetics of levetiracetam during pregnancy, delivery, in the neonatal period and lactation. Epilepsia. 2007;48:111-116.

24. Mazzucchelli I, Onat FY, Ozkara C, et al. Changes in the disposition of oxcarbazepine and its metabolites during pregnancy and puerperium. Epilepsia. 2006;47:504-509. 
25. Ohman I, Sabers A, de Flon P, et al. Pharmacokinetics of topiramate during pregnancy. Epilepsy Res. 2009;87:124-129.

26. Oles KS, Bell WL. Zonisamide concentrations during pregnancy. Ann Pharmacother. 2008;42:1139-1141.

27. Güveli BT, Rosti RÖ, Güzeltaş A, et al. Teratogenicity of antiepileptic drugs. Clin Psychopharmacol Neurosci. 2017;15:19-27.

28. Vajda FJ, O'Brien TJ, Lander CM, et al., Antiepileptic drug combinations not involving valproate and the risk of fetal malformations. Epilepsia. 2016;57:1048-1052.

29. Gerard EE, Meador KJ. An update on maternal use of antiepileptic medications in pregnancy and neurodevelopment outcomes. J Pediatr Genet. 2015;4:94-110.

30. Anderson GD. Pregnancy-related changes in pharmacokinetics: a mechanistic-based approach. Clin Pharmacokinet. 2005;44:989-1008.

31. Ohman I, Vitols S, Tomson T, Pharmacokinetics of gabapentin during delivery, in the neonatal period, and lactation: does a fetal accumulation occur during pregnancy? Epilepsia. 2005;46:1621-1624.

32. Tomson T, Landmark CJ, Battino D. Antiepileptic drug treatment in pregnancy: changes in drug disposition and their clinical implications. Epilepsia. 2013;54:405-414.

33. Pennell PB. Antiepileptic drug pharmacokinetics during pregnancy and lactation. Neurology. 2003;61(suppl 2):S35-S42.

34. Johannessen Landmark C, Farmen AH, Burns ML, et al. Pharmacokinetic variability of valproate during pregnancy - Implications for the use of therapeutic drug monitoring. Epilepsy Res. 2018;141:31-37.

35. Cloyd JC, Lackner TE, Leppik IE. Antiepileptic drugs in the elderly. Pharmacoepidemiology and pharmacokinetics Arch Fam Med. 1994;3:589-598.

36. Crooks J, Stevenson IH. Drug response in the elderly: sensitivity and pharmacokinetic considerations. Age Ageing. 1981;10:73-80.

37. Baftiu A, Feet SA, Larsson PG, et al. Utilisation and polypharmacy aspects of antiepileptic drugs in elderly versus younger patients with epilepsy: A pharmacoepidemiological study of CNS-active drugs in Norway, 2004-2015. Epilepsy Res. 2017;139:35-42. 
38. Wallace SM, Verbeeck RK. Plasma protein binding of drugs in the elderly. Clin Pharmacokinet. 1987;12:41-72.

39. Aronsen KF, Ekelund G, Kindmark CO, et al. Sequential changes of plasma proteins after surgical trauma. Scand J Lab Invest Suppl. 1972;124:127-136.

40. Bowdle TA, Neal GD, Levy RH, et al. Phenytoin pharmacokinetics in burned rats and plasma protein binding of phenytoin in burned patients. $J$ Pharmacol Exp Ther. 1980;213:97-99.

41. Martyn JAJ, Abernethy DR, Greenblatt DJ. Plasma protein binding of drugs after severe burn injury. Clin Pharmacol Ther. 1984;35:535-539.

42. Burger DM, Meenhorst PL, Mulder JW, et al. Therapeutic drug monitoring of phenytoin in patients with acquired immunodeficiency syndrome. Ther Drug Monit. 1994;16:616620.

43. Boggs JG. Elderley patients with systemic disease. Epilepsia. 2001;42(suppl 8):18-23.

44. Perucca E. Free level monitoring of antiepileptic drugs. Clinical usefulness and case studies. Clin Pharmacokinet. 1984;9(suppl 1):71-78.

45. Asconape JJ, Penry JK. Use of antiepileptic drugs in the presence of liver and kidney disease: a review. Epilepsia. 1982;23(suppl 1):S65-S79.

46. Patsalos PN, Froscher W, Pisani $F$, et al. The importance of drug interactions in epilepsy therapy. Epilepsia. 2002;43:365-385.

47. Patsalos PN, Perucca E. Clinically important drug interactions in epilepsy: general features and interactions between antiepileptic drugs. Lancet Neurol. 2003;2:347-356.

48. Patsalos PN. Drug Interactions with the newer antiepileptic drugs (AEDs) - Part 1: Pharmacokinetic and pharmacodynamic interactions between AEDs. Clin Pharmacokin. 2013:52:927-966.

49. Patsalos PN. Drug interactions with the newer antiepileptic drugs (AEDs) - Part 2: Pharmacokinetic and pharmacodynamic interactions between AEDs and drugs used to treat non-epilepsy disorders. Clin Pharmacokin. 2013:52: 1045-1061.

50. Patsalos PN, Perucca E. Clinically important drug interactions in epilepsy: interactions between antiepileptic drugs and other drugs. Lancet Neurol. 2003;2: 473-481. 
51. Perucca E, Albani F, Capovilla G, et al. Recommendations of the Italian League against Epilepsy working group on generic products of antiepileptic drugs. Epilepsia. 2006;47(suppl 5):16-20.

52. Berg MJ, Gross RA, Tomaszewski KJ. Generic substitution in the treatment of epilepsy: case evidence of breakthrough seizures. Neurology. 2008;71:525-530.

53. Fitzgerald CL, Jacobson MP. Generic substitution of levetiracetam resulting in increased breakthrough seizures. Ann Pharmacother. 2011;45:e27.

54. Andermann F, Duh MS, Gosselin A, Paradis PE. Compulsory generic substitution and antiepileptic drugs: High switchback rates to branded compounds compared to other drug classes. Epilepsia. 2007;48:464-469.

55. LeLorier J, Duh MS, Paradis PE, et al. Clinical consequences of genetic substitution of lamotrigine for patients with epilepsy. Neurology. 2008;70:2179-2186.

56. Zachry WM, Doan QD, Smith BJ, et al. Case-controlled analysis of ambulance, emergency room, or inpatient hospital events for epilepsy and antiepileptic drug formulation changes. Epilepsia. 2009;50:493-500.

57. Duh MS, Paradis PE, Latremouille-Viau D, et al. The risks and costs of multiplegeneric substitution of topiramate. Neurology. 2009;72:2122-2129.

58. Ting TY, Jiang W, Lionberger $R$, et al. Generic lamotrigine versus brand-name Lamictal bioequivalence in patients with epilepsy: A field test of the FDA bioequivalence standard. Epilepsia. 2015;56:1415-1424.

59. Privitera MD, Welty TE, Gidal BE, et al. Generic-to-generic lamotrigine switches in people with epilepsy: the randomised controlled EQUIGEN trial. Lancet Neurol. 2016;15:365-372.

60. Klein P, Diaz A, Gasalla T, et al. A review of the pharmacology and clinical efficacy of brivaracetam. Clin Pharmacol. 2018;10:1-22.

61. Patsalos PN, Zugman M, Lake C, et al. Serum protein binding of 25 antiepileptic drugs in a routine clinical setting: A comparison of free non-protein-bound concentrations. Epilepsia. 2017;58:1234-1243.

62. Sargentini-Maier ML, Rolan P, Connell J, et al. The pharmacokinetics, CNS pharmacodynamics and adverse event profile of brivaracetam after single increasing oral doses in healthy males. Br J Clin Pharmacol. 2007;63:680-688. 
63. Sargentini-Maier ML, Espie P, Coquette A, et al. Pharmacokinetics and metabolism of 14C-brivaracetam , a novel SV2A ligand, in healthy subjects. Drug Metab Dispo. 2008;36:36-45.

64. Lacroix B, von Rosenstiel P, Sargentini-Maier ML. Population pharmacokinetics of brivaracetam in patients with partial epilepsy. Epilepsia. 2007;48(Suppl 6):333.

65. Reimers A, Berg JA, Burns ML. et al. Reference ranges for antiepileptic drugs revisited: a practical approach to establish national guidelines. Drug Des Develop Ther. 2018;12:271-280.

66. Rolan P, Sargentini-Maier ML, Pigeolet E, et al. The pharmacokinetics, CNS pharmacodynamics and adverse event profile of brivaracetam after multiple increasing oral doses in healthy men. Br J Clin Pharmacol. 2008;66:71-75.

67. Bertilsson L, Hojer B, Tybring G, et al. Autoinduction of carbamazepine metabolism in children examined by a stable isotope technique. Clin Pharmacol Ther. 1980;127:83-88.

68. Kudriakova TB, Sirota LA, Rozova GI, et al. Autoinduction and steady-state pharmacokinetics of carbamazepine and its metabolites. $\mathrm{Br} \mathrm{J} \mathrm{Clin}$ Pharmacol. 1992;33:611-615.

69. Patsalos PN. A comparative pharmacokinetic study of conventional and chewable carbamazepine in epileptic patients. Br J Clin Pharmacol. 1990; 29:574-577.

70. Patsalos PN. Antiepileptic drug interactions: A clinical guide. London: Springer- Verlag, $3^{\text {rd }}$ Edition, 2016.

71. Stockis $A$, Chanteux $H$, Rosa M, et al. Brivaracetam and carbamazepine interaction in healthy subjects and in vitro. Epilepsy Res. 2015:113:19-27.

72. Aucamp AK. Aspects of pharmacokinetics and pharmacodynamics of benzodiazepines with particular reference to clobazam. Drug Dev Res. 1982; Suppl 1:117-126.

73. Aylett SE, Cross JH, Berry D. Clobazam toxicity in a child with epilepsy related to idiosyncratic metabolism. Dev Med Child Neurol. 2005;48:612615. 
74. Dreifuss FE, Penry JK, Rose SW, et al. Serum clonazepam concentrations in children with absence seizures. Neurology. 1975;25:255-258.

75. Andre M, Boutroy MJ, Dubruc C, et al. Clonazepam pharmacokinetics and therapeutic efficacy in neonatal seizures. Eur J Clin Pharmacol. 1986;30: 585-589.

76. Agurell S, Berlin A, Ferngren H, Hellstrom B. Plasma levels of diazepam after parenteral and rectal administration. Epilepsia. 1975;16:277-283.

77. Meberg A, Langslet A, Bredesen JE, Lunde PKM. Plasma concentration of diazepam and $\mathrm{N}$-desmethyldiazepam in children after a single rectal or intramuscular dose. Eur $J$ Clin Pharmacol. 1978;12:273-276.

78. Cloyd JC, Lalonde RL, Beniak TE, et al. A single blind crossover comparison of the pharmacokinetic and cognitive effects of a new diazepam rectal gel with intravenous diazepam. Epilepsia. 1998;39:520-526.

79. Schmidt D. Benzodiazepines: diazepam. In: Levy RH, Mattson RH, Meldrum BS, eds. Antiepileptic drugs, $4^{\text {th }}$ ed. New York:Raven Press, 1995:705-724. 80. Dhillon S, Richens A. Valproic acid and diazepam interaction in vivo. $\mathrm{Br} J$ Clin Pharmacol. 1982;13:553-560.

81. Shorvon S. Status epilepticus: its clinical features and treatment in children and adults. Cambridge University Press: Cambridge; 1994.

82. Hallstrom C, Lader MH. Diazepam and N-desmethyldiazepam concentrations in saliva, plasma and CSF. Br J Clin Pharmacol. 1980;9:333-339.

83. Almeida L, Soares-da-Silva P. Safety, tolerability and pharmacokinetic profile of BIA 2-093, a novel putative antiepileptic, in a rising multiple-dose study in young healthy humans. J Clin Pharmacol. 2004;44:906-918.

84. Buchanan RA, Kinkel AW, Smith TC. The absorption and excretion of ethosuximide. Int J Clin Pharmacol. 1973;7:213-218.

85. Eadie MJ, Tyrer JH, Smith GA, et al. Pharmacokinetics of drugs used for petit mal 'absence' epilepsy. Clin Exp Neurol. 1977;14:172-183.

86. Shumaker RC, Fantel C, Kelton E, et al. Evaluation of the elimination of (14C) felbamate in healthy men. Epilepsia. 1990;31:642. 
87. Sachdeo RC, Kramer LD, Rosenberg A, et al. Felbamate monotherapy: controlled trial in patients with partial onset seizures. Ann Neurol. 1992;32: 385-392.

88. Vollmer KO, von Hodenberg A, Kolle EU. Pharmacokinetics and metabolism of gabapentin in rat, dog and man. Azneimittelforschung. 1986;36:830-839.

89. Johannessen Landmark C, Patsalos PN. Drug interactions involving the new second- and third-generation antiepileptic drugs. Expert Rev Neurother. 2010;10:119-140.

90. Doty P, Rudd GD, Stoehr T, Thomas D. Lacosamide. Neurotherapeutics. 2007; 4:145-148.

91. Patsalos PN, Berry DJ. Pharmacotherapy of the third-generation AEDs: Lacosamide, retigabine and eslicarbazepine acetate. Exp Opin Pharmacother. 2012;13:699-715.

92. Cawello W, Stockis A, Andreas JO, et al. Advances in epilepsy treatment: lacosamide pharmacokinetic profile. Ann N Y Acad Sci. 2014;1329:18-32.

93. Contin M, Albani F. Riva R, et al. Lacosamide therapeutic monitoring in patients with epilepsy: effects of concomitant antiepileptic drugs. Ther Drug Monit. 2013;35:849-852.

94. Markoula S, Teotonio R, Ratnaraj N, et al. Lacosamide serum concentrations in adult patients with epilepsy: The influence of gender, age, dose and concomitant antiepileptic drugs. Ther Drug Monit. 2014;36:494498.

95. Svendsen T, Brodtkorb E, Baftiu A, et al. Therapeutic drug monitoring of lacosamide in Norway: Focus on pharmacokinetic variability, efficacy and tolerability. Neurochem Res. 2017;42:2077-2083.

96. Brandt C, Bien CG, Helmer R, et al. Assessment of correlations of lacosamide concentrations in saliva and serum in patients with epilepsy. Epilepsia. 2018;59:e34e39.

97. Rambeck B, Wolf P. Lamotrigine clinical pharmacokinetics. Clin Pharmacokinet. 1993;5:433-443. 
98. May TW, Rambeck B, Jurgens U. Serum concentrations of lamotrigine in epileptic patients: the influence of dose and comedication. Ther Drug Monit. 1996;18:523-531.

99. Patsalos PN. Pharmacokinetic profile of levetiracetam: toward ideal characteristics. Pharmacol Ther. 2000;85:77-85.

100. Patsalos PN, Ghattaura S, Ratnaraj N, et al. In situ metabolism of levetiracetam in blood of patients with epilepsy. Epilepsia. 2006;47:18181821.

101. Leppik IE, Rarick JO, Walczak TS, et al. Effective levetiracetam doses and serum concentrations: age effects. Epilepsia. 2002;43:240.

102. Patsalos PN, Elyas AA, Zakrzewska JM. Protein binding of oxcarbazepine and its primary active metabolite, 10-hydroxycarbazepine, in patients with trigeminal neuralgia. Eur J Clin Pharmacol. 1990;39:413-415.

103. Volosov A, Xiaodong S, Perucca E, et al. Enantioselective pharmacokinetics of 10-hydroxycarbazepine after oral administration of oxcarbazepine to healthy Chinese subjects. Clin Pharmacol Ther. 1999;66:547-553.

104. Wellington K, Goa KL. Oxcarbazepine-an update of its efficacy in the management of epilepsy. CNS Drugs. 2001;15:137-163.

105. Patsalos PN. The clinical pharmacology profile of the new antiepileptic drug perampanel: A novel non-competitive AMPA receptor antagonist. Epilepsia. 2015;56:12-27.

106. Nelson E, Powell JR, Conrad K, et al. Phenobarbital pharmacokinetics and bioavailability in adults. J Clin Pharmacol. 1982;22:141-148.

107. Wilensky AJ, Friel PN, Levy $\mathrm{RH}$, et al. Kinetics of phenobarbital in normal subjects and epileptic patients. Eur J Clin Pharmacol. 1982;23:87-92.

108. Richens A. Clinical pharmacokinetics of phenytoin. Clin Pharmacokinet. 1979;4:153-169.

109. Neurvonen PJ. Bioavailability of phenytoin: clinical pharmacokinetic and therapeutic implications Clin Pharmacokinet. 1979;4:91-103. 
110. Shorvon SD. Pyrrolidine derivatives. Lancet. 2001;358:1885-1892.

111. Ben-Menachem E. Pregabalin pharmacology and its relevance to clinical practice. Epilepsia. 2004;45:13-18.

112. Gallagher BB, Baumel IP, Mattson RH. Metabolic disposition of primidone and its metabolites in epileptic subjects after single and repeated administration. Neurology. 1972;22:1186-1192.

113. Cardot JM, Lecaillon JB, Czendlik C, et al. The influence of food on the disposition of the antiepileptic rufinamide in healthy volunteers. Biopharm Drug Dispos. 1998;19:259-262.

114. Perucca E, Cloyd J, Critchley D, et al. Rufinamide: clinical pharmacokinetics and concentration-response relationships in patients with epilepsy. Epilepsia. 2008;49:1123-1141.

115. Wheless JW, Vazquez B. Rufinamide: a novel broad-spectrum antiepileptic drug. Epilepsy Curr. 2010;10:1-6.

116. Levy RH, Loiseau P, Guyot M, et al. Stiripentol kinetics in epilepsy: nonlinearity and interactions. Clin Pharmacol Ther. 1984;36:661-669.

117. Chiron C. Stiripentol. Neurotherapeutics. 2007;4:123-125.

118. Levy $\mathrm{RH}$, Lin HS, Blehaut HM, et al. Pharmacokinetics of stiripentol in normal man: evidence of nonlinearity. J Clin Pharmacol. 1983;23:523-533.

119. Moreland TA, Astoin J, Lepage F. The metabolic fate of stiripentol in man. Drug Metab Dispos. 1986;14:654-662.

120. Chiron C, Marchand MC, Tran A, et al. Stiripentol in severe myoclonic epilepsy in infancy: a randomized placebo-controlled syndrome-dedicated trial. STICLO study group. Lancet. 2000;356:1638-1642.

121. Farwell JR, Anderson GD, Kerr BM, et al. Stiripentol in atypical absence seizures in children: an open trial. Epilepsia. 1993;34:305-311.

122. May TW, Korn-Merker E, Rambeck B, et al. Pharmacokinetics of sulthiame in epileptic patients. Ther Drug Monit. 1994;16:251-257. 
123. Gustavson LE, Mengel HB. Pharmacokinetics of tiagabine, a $\gamma-$ aminobutyric acid-uptake inhibitor in healthy subjects after single and multiple doses. Epilepsia. 1995;36:605-611.

124. Patsalos PN, Elyas AA, Ratnaraj N, et al. Concentration-dependent displacement of tiagabine by valproic acid. Epilepsia. 2002; 43: 143.

125. Ulthman BM, Rowan JA, Ahmann PA, et al. Tiagabine for complex partial seizures. A randomized, add-on, dose-response trial. Arch Neurol. 1998;55: 56-62.

126. Patsalos PN. The pharmacokinetic profile of topiramate. Rev Contemp Pharmacother. 1999;10:155-162.

127. Sachdeo RC, Sachdeo SK, Walker SA, et al. Steady state pharmacokinetics of topiramate and carbamazepine in patients with epilepsy during monotherapy and concomitant therapy. Epilepsia. 1996;37:774-780.

128. Britzi M, Perucca E, Soback S, et al. Pharmacokinetic and metabolic investigation of topiramate disposition in healthy subjects in the absence and in the presence of enzyme induction by carbamazepine. Epilepsia. 2005;46:378-384.

129. Cramer JA, Mattson $\mathrm{RH}$, Bennett DM, et al. Variable free and total valproic acid concentrations in sole- and multi-drug therapy. Ther Drug Monit. 1986;8:411-415.

130. Perucca E, Gatti G, Frigo GM, et al. Disposition of sodium valproate in epileptic patients. Br J Clin Pharmacol. 1978;5:495-499.

131. Dwivedi R, Gupta YK, Singh M, et al. Correlation of saliva and serum free valproic acid concentrations in persons with epilepsy. Seizure. 2015;25:187-190.

132. Haegele KD, Huebert ND, Ebel M, et al. Pharmacokinetics of vigabatrin: implications of creatinine clearance. Clin Pharmacol Ther. 1988;44:558-565.

133. Patsalos PN, Duncan JN. The pharmacology and pharmacokinetics of vigabatrin. Rev Contemp Pharmacother. 1995;6:447-456.

134. Arteaga R, Harranz JL, Valdizan EM, et al. Gamma-vinyl-GABA (vigabatrin): relationship between dosage, plasma concentrations, platelet 
GABA-transaminase inhibition, and seizure reduction in children. Epilepsia. 1992;33:923-931.

135. Ito $\mathrm{T}$, Yamaguchi $\mathrm{T}$, Miyizaki $\mathrm{H}$, et al. Pharmacokinetic studies of AD-810, a new antiepileptic compound. Arzneimittelforschung. 1982;32:1581-1586.

136. Matsumoto K, Miyazaki H, Fujii T, et al. Absorption, distribution and excretion of 3-(sulfamoyl[14C]methyl)-1,2-benziosoxazole (AD-810) in rats, dogs and monkeys and AD-810 in men. Arzneimettelforschung. 1983;33: 961-968.

137. Kochak GM, Page JG, Buchanan RA, et al. Steady-state pharmacokinetics of zonisamide, an antiepileptic agent for treatment of refractory complex partial seizures. J Clin Pharmacol. 1998;38:166-171.

138. Mimaki T. Clinical pharmacology and therapeutic drug monitoring of zonisamide. Ther Drug Monit. 1998;20:593-697.

139. Berent S, Sackellares JC, Giordani B, et al. Zonisamide (Cl-912) and cognition: results from preliminary study. Epilepsia. 1987;28:61-67.

140. O'Neal CL, Crouch DJ, Rollins DE, et al. Correlation of saliva codeine concentrations with plasma concentrations after oral codeine administration. J Anal Toxicol. 1999;23:452-459.

141. Crouch DJ. Oral fluid collection: the neglected variable in oral fluid testing. Forensic Sci Int. 2005;150:165-173.

142. Dickinson RG, Hooper WD, King AR, et al. Fallacious results from measuring salivary carbamazepine concentrations. Ther Drug Monit. 1985;7:41-45.

143. . Ayers GJ, Burnett D. Drug formulation and salivary phenytoin measurements. Lancet. 1977;1:656.

144. Lins RL, Otoul C, De Smedy F, et al. Comparison of plasma and saliva concentrations of levetiracetam following administration orally as a tablet and as a solution in healthy adult volunteers. Int J Clin Pharmacol Ther. 2007;45:47-54.

145. Edelbroek PM, van der Heijden J, Stolk LML. Dried blood spot methods in therapeutic drug monitoring: methods, assays, and pitfalls. Ther Drug Monit. 2009;31:327-336. 
146. Sadones N, Van Bever E, Van Bortel L, et al. Dried blood spot analysis of gabapentin as a valid alternative for serum: a bridging study. J Pharm Biomed Anal. 2017;132:72-76.

147. Linder C, Hansson A, Sadek S, et al. Carbamazepine, lamotrigine, levetiracetam and valproic acid in dried blood spots with liquid chromatography tandem mass spectrometry; method development and validation. J Chromatogr B Analyt Technol Biomed Life Sci. 2018;1072:116-122.

148. Hahn RZ, Antunes MV, Costa Arnhold P, et al. Determination of topiramate in dried blood spots using single-quadrupole gas chromatography-mass spectrometry after flash methylation with trimethylanilinium hydroxide. J Chromatogr B Analyt Technol Biomed Life Sci. 2017;1046:131-137.

149. Linder C, Wide K, Walander M, et al. Comparison between dried blood spot and plasma sampling for therapeutic drug monitoring of antiepileptic drugs in children with epilepsy: A step towards home sampling. Clin Biochem. 2017;50:418-424.

150. Kolocouri F, Dotsikas Y, Loukas YL. Dried plasma spots as an alternative sample collection technique for the quantitative LC-MS/MS determination of gabapentin. Anal Bioanal Chem. 2010;398:1339-1347.

151. Wilhelm AJ, den Burger JCG, Swart EL. Therapeutic drug monitoring by dried blood spot: Progress to date and future directions. Clin Pharmacokinet. 2014;53:961-973.

152. Kloosterboer SM, de Winter BCM, Bahmany S, et al. Dried blood spot analysis for therapeutic drug monitoring of antipsychotics: Drawbacks of Its clinical application. Ther Drug Monit. 2018 doi:10.1097/FTD 00000000502.

153. Patsalos PN. Personal Communication. 2018.

154. Bailey DN, Coffee JJ, Briggs JR. Stability of drug concentrations in plasma stored in serum separator blood collection tubes. Ther Drug Monit. 1988;10:352-354.

155. Dasgupta A, Yared MA, Wells A. Time-dependent absorption of therapeutic drugs by the gel of Greiner Vacuette blood collection tube. Ther Drug Monit. 2000;22:427431.

156. O'Keane MP, Cunninmgham SK. Evaluation of three different specimen types (serum, plasma lithium heparin and serum gel separator) for analysis of certain 
analytes: clinical significance of differences in results and efficiency in use. Clin Chem Lab Med. 2006;44:662-668.

157. Mauro LS, Mauro VF. Effect of separator tubes on free and total phenytoin and carbamazepine serum concentrations. Ther Drug Monit. 1991;13:240-243.

158. Dasgupta A, Dean R, Saldana S, et al. Absorption of therapeutic drugs by barrier gels in serum separator blood collection tubes. Volume- and time-dependent reduction in total and free drug concentrations. Am J Clin Pathol. 1994;101:456-461.

159. Rosenthal $\mathrm{E}$, Hoffer $\mathrm{E}$, Ben-Aryeh $\mathrm{H}$, et al. Use of saliva in home monitoring of carbamazepine levels. Epilepsia. 1995;36:72-74.

160. Tennison MB, Miles MV, Ali I, et al. Salivary monitoring of lamotrigine and gabapentin. Epilepsia. 1999;40(Supp. 7):144.

161. Tennison M, Ali I, Miles MV, et al. Feasibility and acceptance of salivary monitoring of antiepileptic drugs via the US Postal Service. Ther Drug Monit. 2004;26:295-299.

162. Jones MD, Ryan M, Miles MV, et al. Stability of salivary concentrations of the newer antiepileptic drugs in the postal system. Ther Drug Monit. 2005; 27:576-579.

163. Bondareva I. Individualizing antiepileptic therapy for patients. In: Individualized Drug Therapy for Patients: Basic Foundations, Relevant Software, and Clinical Applications, eds. Jelliffe RW, Neely M. Academic Press, 2017 pp 327-371.

164. Jelliffe R, Bayard D, Neely M. Monitoring each patient optimally: When to obtain the best samples for therapeutic drug monitoring. In: Individualized Drug Therapy for Patients: Basic Foundations, Relevant Software, and Clinical Applications, eds. Jelliffe RW, Neely M. Academic Press, 2017, pp 91-102.

165. Chang Y, Yang LY, Zhang MC, et al., Correlation of the UGT1A4 gene polymorphism with serum concentration and therapeutic efficacy of lamotrigine in Han Chinese of Northern China. Eur J Clin Pharmacol. 2014;70:941-946.

166. Gulcebi MI, Ozkaynakci A, Goren MZ, et al. The relationship between UGT1A4 polymorphism and serum concentrations of lamotrigine in patients with epilepsy. Epilepsy Res. 2011;95:1-8.

167. Liu L, Zhao L, Wang Q, et al. Influence of valproic acid concentration and 
polymorphism of UGT1A4 ${ }^{\star} 3$, UGT2B7-161C $>$ T and UGT2B7 ${ }^{\star} 2$ on serum concentration of lamotrigine in Chinese epileptic children. Eur J Clin Pharmacol. 2015;71:1341-1347.

168. Singkham $N^{1}$, Towanabut S, Lertkachatarn S, et al. Influence of the UGT2B7 $161 \mathrm{C}>\mathrm{T}$ polymorphism on the population pharmacokinetics of lamotrigine in Thai patients. Eur J Clin Pharmacol. 2013;69:1285-1291.

169. Tassaneeyakul W, Prabmeechai N, Sukasem C, et al. Associations between HLA class I and cytochrome P450 2C9 genetic polymorphisms and phenytoin-related severe cutaneous adverse reactions in a Thai population. Pharmacogenet Genomics. 2016;26:225-234.

170. Shi YW, Min FL, Zhou D, et al. HLA-A*24:02 as a common risk factor for antiepileptic drug-induced cutaneous adverse reactions. Neurology. 2017;88:2183-2191.

171. Ito $A$, Shimada $H$, Ishikawa $K$, et al. Association between HLA-DRB1*0405, DQB1 ${ }^{*} 0401$ and -DQA1*0303 alleles and lamotrigine-induced cutaneous adverse drug reactions. A pilot case-control study from Japan. J Affect Disord. 2015;179:47-50.

172. Chouchi M, Kaabachi W, Tizaoui K, et al. The HLA-B*15:02 polymorphism and Tegreto ${ }^{\circledR}{ }^{\circledR}$-induced serious cutaneous reactions in epilepsy: An updated systematic review and meta-analysis. Rev Neurol (Paris). 2018 Apr 20. pii: S00353787(16)30339-3. doi: 10.1016/j.neurol.2017.11.006. [Epub ahead of print] 
Table 1: Introduction of antiepileptic drugs in the United Kingdom

\begin{tabular}{|l|l|}
\hline Drug & Year of Introduction \\
\hline & \\
\hline Phenobarbital & 1912 \\
\hline Phenytoin & 1938 \\
\hline Primidone & 1952 \\
\hline Ethosuximide & 1960 \\
\hline Carbamazepine & 1963 \\
\hline Diazepam & 1973 \\
\hline Valproate & 1974 \\
\hline Clonazepam & 1974 \\
\hline Clobazam & 1982 \\
\hline Vigabatrin & 1989 \\
\hline Lamotrigine & 1991 \\
\hline Gabapentin & 1993 \\
\hline Felbamate & 1993 \\
\hline Topiramate & 1995 \\
\hline Fosphenytoin & 1996 \\
\hline Piracetam & 1997 \\
\hline Tiagabine & 1998 \\
\hline Oxcarbazepine & 2000 \\
\hline Levetiracetam & 2000 \\
\hline Pregabalin & 2004 \\
\hline Zonisamide & 2005 \\
\hline Rufinamide & 2007 \\
\hline Stiripentol & 2007 \\
\hline Lacosamide & 2008 \\
\hline Eslicarbazepine acetate & 2009 \\
\hline Perampanel & 2012 \\
\hline Brivaracetam & 2016 \\
\hline & \\
\hline
\end{tabular}




\begin{tabular}{|c|c|c|}
\hline & Indication & Comment \\
\hline 1 & $\begin{array}{l}\text { After initialization of AED } \\
\text { treatment or after dose } \\
\text { adjustment }\end{array}$ & $\begin{array}{l}\text { This allows the pursuance of a preselected reference } \\
\text { range for the individual patient } \\
\text { Always measure blood concentrations before a dose } \\
\text { change and after achievement of steady state at new } \\
\text { dose }\end{array}$ \\
\hline 2 & $\begin{array}{l}\text { Upon achievement of optimum } \\
\text { desired clinical response }\end{array}$ & $\begin{array}{l}\text { Seizure freedom is the optimum outcome but for } \\
\text { many patients optimum seizure control with } \\
\text { minimal adverse effects is more readily achieved. } \\
\text { This allows for the "individual therapeutic range" to } \\
\text { be established } \\
\text { In order to establish an individual's therapeutic } \\
\text { concentration, two separate determinations should } \\
\text { be undertaken 2-4 months apart in order to estimate } \\
\text { the extent of any variability }\end{array}$ \\
\hline 3 & $\begin{array}{l}\text { To determine the magnitude of } \\
\text { a dose change }\end{array}$ & $\begin{array}{l}\text { This is particularly important for AEDs that show } \\
\text { dose-dependent pharmacokinetics (e.g. phenytoin, } \\
\text { carbamazepine, valproate, gabapentin, stiripentol } \\
\text { and rufinamide) } \\
\text { Always measure blood concentrations before a dose } \\
\text { change and after achievement of steady-state at new } \\
\text { dose }\end{array}$ \\
\hline 4 & When toxicity is difficult to & Concentration-related AED toxicity is more readily \\
\hline
\end{tabular}




\begin{tabular}{|c|c|c|}
\hline & $\begin{array}{l}\text { differentially diagnose or when } \\
\text { toxicity is difficult to assess } \\
\text { clinically }\end{array}$ & $\begin{array}{l}\text { identified and is particularly helpful when young } \\
\text { children or patients with mental disability are being } \\
\text { evaluated } \\
\text { Elevated plasma concentrations of many AEDs can } \\
\text { exacerbate seizures and TDM will assist the } \\
\text { differential diagnosis in the case of a known } \\
\text { epileptic patient admitted with seizures }\end{array}$ \\
\hline 5 & $\begin{array}{l}\text { When seizures persist despite } \\
\text { the prescribing of an } \\
\text { adequate/typical dosage }\end{array}$ & $\begin{array}{l}\text { This may identify a fast metabolizer or a patient that } \\
\text { is non-complying with their AED medication }\end{array}$ \\
\hline 6 & $\begin{array}{l}\text { When pharmacokinetic } \\
\text { variability is expected. }\end{array}$ & $\begin{array}{l}\text { This entails a significant category of patients and } \\
\text { includes children, the elderly, during pregnancy, } \\
\text { hepatic disease, renal disease, various pathologies, } \\
\text { post-surgery and drug-drug pharmacokinetic } \\
\text { interactions. }\end{array}$ \\
\hline 7 & $\begin{array}{l}\text { When a formulation change is } \\
\text { to occur }\end{array}$ & $\begin{array}{l}\text { This includes immediate-release to sustained-release } \\
\text { formulations and brand to generic and generic to } \\
\text { generic switches. } \\
\text { Always measure blood concentrations before a } \\
\text { formulation change and after achievement of steady } \\
\text { state with the new formulation }\end{array}$ \\
\hline 8 & $\begin{array}{l}\text { The clinical response has } \\
\text { unexpectantly changed }\end{array}$ & $\begin{array}{l}\text { The cause of the change could be readily identified } \\
\text { as it could be the consequence of many reasons }\end{array}$ \\
\hline 9 & Poor compliance suspected & $\begin{array}{l}\text { Recent non-compliance can be readily identified. } \\
\text { However, long-term compliance or variable } \\
\text { compliance cannot be identified. }\end{array}$ \\
\hline
\end{tabular}


Table 3: Pharmacokinetic parameters and serum reference ranges for the various antiepileptic drugs (AEDs) prescribed as monotherapy to adults.

\begin{tabular}{|c|c|c|c|c|c|c|c|c|}
\hline & \multirow{2}{*}{$\begin{array}{l}\text { Time to } \\
\text { steady-state } \\
\text { (days) }\end{array}$} & \multirow{2}{*}{\begin{tabular}{|l} 
Plasma \\
protein \\
binding $(\%)$ \\
I
\end{tabular}} & \multirow[t]{2}{*}{$\begin{array}{l}\text { Half-life } \\
\text { (h) }\end{array}$} & \multirow{2}{*}{$\begin{array}{l}\text { Pharmacologically } \\
\text { active metabolites that } \\
\text { also need monitoring }\end{array}$} & \multicolumn{2}{|c|}{$\begin{array}{l}\text { Plasma reference range } \\
\text { a }\end{array}$} & \multicolumn{2}{|c|}{ Monitoring useful of: } \\
\hline & & & & & $\mathrm{mg} / \mathrm{L}$ & $\mu \mathrm{mol} / \mathrm{L}$ & Saliva & $\begin{array}{l}\text { Plasma free } \\
\text { fraction }\end{array}$ \\
\hline $\begin{array}{l}\text { Brivaracetam } \\
\text { (Briviact } ®)\end{array}$ & $1-2$ & 35 & $7-8$ & & $0.2-2$ & $1-10$ & Yes & No \\
\hline $\begin{array}{l}\text { Clobazam } \\
\text { (Frisium } ®)\end{array}$ & $7-10^{c}$ & 90 & $10-30$ & $\begin{array}{l}\text { N-Desmethyl- } \\
\text { clobazam }\end{array}$ & $\begin{array}{l}0.03-0.3 \\
0.3-3.0^{\mathbf{d}}\end{array}$ & $\begin{array}{l}0.1-1.0 \\
1.0-10.5^{\mathrm{d}}\end{array}$ & Yes & No \\
\hline $\begin{array}{l}\text { Clonazepam } \\
\text { (Rivotril®) }\end{array}$ & $3-10$ & 90 & $17-56$ & & $0.02-0.07$ & $0.06-0.22$ & No & No \\
\hline $\begin{array}{l}\text { Ethosuximide } \\
(\text { Emeside } ®)\end{array}$ & $8-12$ & 22 & $40-60$ & & $40-100$ & $283-708$ & Yes & No \\
\hline $\begin{array}{l}\text { Felbamate } \\
(\text { Felbatol®) }\end{array}$ & $3-5$ & 48 & $16-22$ & Page 60 of 65 & $30-60$ & $126-252$ & Yes & No \\
\hline Gabapentin & $1-2$ & 0 & $5-9$ & & $2-20$ & $12-117$ & Yes & No \\
\hline
\end{tabular}




\begin{tabular}{|c|c|c|c|c|c|c|c|c|}
\hline (Neurontin $®)$ & & & & & & & & \\
\hline $\begin{array}{l}\text { Lacosamide } \\
\text { (Vimpat } ®)\end{array}$ & $2-3$ & 14 & $12-14$ & & $10-20$ & $40-80$ & Yes & No \\
\hline $\begin{array}{l}\text { Lamotrigine } \\
\text { (Lamictal@) }\end{array}$ & $3-8$ & 66 & $15-35$ & & $2.5-15$ & $10-59$ & Yes & Yes \\
\hline $\begin{array}{l}\text { Oxcarbazepine }^{\mathbf{g}} \\
\text { (Trileptal®) }\end{array}$ & $2-3$ & 40 & $8-15$ & $\begin{array}{l}10- \\
\text { hydroxycarbazepine }\end{array}$ & $3-35$ & $12-139$ & Yes & No \\
\hline $\begin{array}{l}\text { Perampanel } \\
\text { (Fycompa®) }\end{array}$ & $10-19$ & 98 & 48 & & $0.18-0.98$ & $0.50-2.74$ & $\mathrm{NE}$ & Yes \\
\hline $\begin{array}{l}\text { Phenytoin } \\
\text { (Epanutin } ®)\end{array}$ & $6-21$ & 92 & $30-100^{h}$ & & $10-20$ & $40-79$ & Yes & Yes \\
\hline $\begin{array}{l}\text { Pregabalin } \\
\text { (Lyrica®) }\end{array}$ & $1-2$ & 0 & $5-7$ & & $2-8$ & $13-50$ & $\mathrm{NE}$ & No \\
\hline $\begin{array}{l}\text { Primidone } \\
\left.\text { (Mysoline }{ }^{\circledR}\right)\end{array}$ & $2-5$ & 33 & $7-22$ & Phenobarbital & $5-10^{j}$ & $23-46$ & Yes & No \\
\hline $\begin{array}{l}\text { Rufinamide } \\
\text { (Inovelon®) }\end{array}$ & $1-2$ & 28 & $6-10$ & & $30-40$ & $126-168$ & Yes & No \\
\hline
\end{tabular}

Page 61 of 65 


\begin{tabular}{|c|c|c|c|c|c|c|c|}
\hline (Diacomit@) & & & & & & & \\
\hline $\begin{array}{l}\text { Tiagabine } \\
\text { (Gabitril@) }\end{array}$ & $1-2$ & 98 & $5-9$ & $0.02-0.2$ & $0.05-0.53$ & $\mathrm{NE}$ & No \\
\hline $\begin{array}{l}\text { Topiramate } \\
\text { (Topamax } ®)\end{array}$ & $4-7$ & 20 & $20-30$ & $5-20$ & $15-59$ & Yes & No \\
\hline $\begin{array}{l}\text { Vigabatrin } \\
\text { (Sabril@) }\end{array}$ & $1-2$ & 17 & $5-8$ & $0.8-36$ & $6-279$ & $\mathrm{NE}$ & No \\
\hline $\begin{array}{l}\text { Zonisamide } \\
\text { (Zonegran®) }\end{array}$ & $9-12$ & 40 & $50-70$ & $10-40$ & $47-188$ & Yes & No \\
\hline
\end{tabular}

Table 3: Pharmacokinetic parameters and serum reference ranges for the various antiepileptic drugs (AEDs) prescribed as monotherapy to adults.

$a=$ for clarity values can be rounded up or down by laboratory; $b=$ refers to patients on chronic therapy after autoinduction has completed - values are much longer after a single dose; $\mathrm{c}=$ includes time to steady-state for active metabolite $\mathrm{N}$-desmethyl-clobazam; $d=$ refers to values for active metabolite $\mathrm{N}$-desmethyl-clobazam; $\mathrm{e}=$ all values refer to the active metabolite eslicarbazepine; $\mathrm{f}=$ the reference range is that quoted for the active metabolite of oxcarbazepine namely10-hydroxycarbazepine because the two molecules are identical; $\mathrm{g}=\mathrm{all}$ values refer to the active metabolite 10-hydroxycarbazepine; $\mathrm{h}=$ elimination is saturable so that half-life increases with increasing plasma concentration; $\mathrm{i}=$ not established; $\mathrm{j}=$ during treatment with primidone both primidone and the pharmacologically active metabolite phenobarbital should be monitored; $\mathrm{k}=$ There are clinical settings where monitoring of carbamazepine-epoxide, in addition to 
carbamazepine, is warranted, particularly when a co-medication occurs with an inhibitor of carbamazepine-epoxide metabolism; $1=$ data from Patsalos et al., 2017; $\mathrm{m}=$ Plasma protein binding of valproic acid is concentration dependent and therefore variable. 
Table 4: Antiepileptic drug (AED) therapeutic drug monitoring (TDM): Guidelines for optimum clinical use

The laboratory undertaking TDM must be accredited and have appropriate quality control procedures in place and it is preferable that the laboratory is able to quantitate all AEDs that are licensed for clinical use, including any known pharmacologically active metabolites

Knowledge of the pharmacokinetic and pharmacodynamic characteristics of the AED to be monitored is essential

Concentration measurements should only be undertaken when there is a clear clinical question The matrix of choice is presently blood (plasma or serum). However, there are clinical settings, and also by patient choice, that saliva is employed. Free non-protein concentration measurement is also indicated in certain settings

As a rule of thumb concentrations should be measured at steady state. The exceptions to this are in the event of overdose, suspected toxicity or in patients with a diagnosis of status epilepticus It is imperative that sampling times are standardized and in most clinical settings it is best that a sample is taken after an overnight fast and immediately before the morning dose. Overall sampling before the next dose is recommended. In an out-patient setting this is not always possible; therefore, the sampling time relative to dose ingestion should be recorded so as to aid interpretation of the measured concentration

Diurnal changes in concentrations, particularly for AEDs with short half-lives (<12 hours), can complicate interpretation of concentration measurements. Therefore, interpretation of concentration measurements must take into consideration the interval since last dose ingestion and the known pharmacokinetic characteristics of the AED

The clinician must be made aware that AED reference ranges quoted by a laboratory are population values and that a significant minority of patients may require concentrations above or below these ranges. This information needs also to be communicated to the patient or his/her carers The concept of the "individual therapeutic concentration" should be applied to as many patients as possible 
Interpretation of AED concentrations requires understanding of situations which may alter the relationship between AED concentrations and clinical response. For example, severity and type of epilepsy, clinical (hepatic or renal disease) and physiological (pregnancy, old age) conditions that alter plasma protein binding and the presence of pharmacologically active metabolites. Additional considerations for those patients on polytherapy regimens include the increased presence of pharmacologically active metabolites, displacement protein binding interactions and pharmacodynamic interactions with concurrently administered drugs (both AEDs and drugs used to treat concurrent diseases)

Whilst adjusting drug dosages to achieve plasma concentrations within the reference range should be the initial goal, until the clinical response to therapy can be assessed, it should be emphasized that it is important to treat the patient and not the blood concentration. Clinical decisions should also be based on patient history, including laboratory information and clinical signs and symptoms 